DRAFT VERSION OCTOBER 28, 2018

Preprint typeset using $\mathrm{LT}_{\mathrm{E}} \mathrm{X}$ style emulateapj v. 08/22/09

\title{
OBSCURING ACTIVE GALACTIC NUCLEI WITH NUCLEAR STARBURST DISKS
}

\author{
D. R. BALLANTYNE \\ Department of Physics, The University of Arizona, 1118 East 4th Street, Tucson, AZ 85721; drb@ physics.arizona.edu \\ Draft version October 28, 2018
}

\begin{abstract}
We assess the potential of nuclear starburst disks to obscure the Seyfert-like AGN that dominate the hard $\mathrm{X}$-ray background at $z \sim 1$. Over 1200 starburst disk models, based on the theory developed by Thompson et al., are calculated for five input parameters: the black hole mass, the radial size of the starburst disk, the dustto-gas ratio, the efficiency of angular momentum transport in the disk, and the gas fraction at the outer disk radius. We find that a large dust-to-gas ratio, a relatively small starburst disk, a significant gas mass fraction, and efficient angular momentum transport are all important to produce a starburst disk that can potentially obscure an AGN. The typical maximum star-formation rate in the disks is $\sim 10 \mathrm{M}_{\odot} \mathrm{yr}^{-1}$. Assuming no mass-loss due to outflows, the starburst disks feed gas onto the black hole at rates sufficient to produce hard $\mathrm{X}$-ray luminosities of $10^{43}-10^{44} \mathrm{erg} \mathrm{s}^{-1}$. The starburst disks themselves should be detectable at mid-infrared and radio wavelengths; at $z=0.8$, the predicted fluxes are $\sim 1 \mathrm{mJy}$ at $24 \mu \mathrm{m}$ and $\sim 10-30 \mu \mathrm{Jy}$ at $1.4 \mathrm{GHz}$. Thus, we predict a large fraction of radio/X-ray matches in future deep radio surveys. Unfortunately, both the $24 \mu \mathrm{m}$ and radio fluxes are comparable to those expected from the central AGN. In contrast, the starburst disks should be easily distinguished from AGN in future $100 \mu \mathrm{m}$ surveys by the Herschel Space Observatory with expected fluxes of $\sim 5 \mathrm{mJy}$. Any AGN-obscuring starbursts will be associated with hot dust, independent of AGN heating, resulting in observable signatures for separating galactic and nuclear star-formation. This may be an explanation for the small observed $L_{2-10 \mathrm{keV}} / \nu L_{\nu}(6 \mu \mathrm{m})$ ratios observed from both $z \sim 0$ and $z \sim 1$ AGN. Finally, because of the competition between gas and star-formation, nuclear starbursts will be associated with lower-luminosity AGN. Thus, this phenomenon is a natural explanation for the observed decrease in the fraction of obscured AGN with luminosity.
\end{abstract}

Subject headings: galaxies: active — galaxies: evolution — galaxies: formation — galaxies: Seyfert — galaxies: starburst $-\mathrm{X}$-rays: diffuse background

\section{INTRODUCTION}

All Active Galactic Nuclei (AGN) are powered by accretion onto a central supermassive black hole (Lynden-Bell 1969; Shakura \& Sunyaev 1973; Pringle 1981; Balbus 2003). However, the observational manifestation of this accretion can vary dramatically from object to object. For example, the spectral energy distributions (SED) of rapidly accreting quasars (Elvis et al. 1994) are very different from the Galactic Center source Sgr A* (Melia \& Falcke 2001), or the black hole at the center of M87 (Ho 1999). In this case, the different observational properties are a result of the two distinct mechanisms through which energy is liberated in radiatively efficient (for the quasars) and inefficient (for Sgr A* and M87) accretion flows. Another important parameter is the orientation of the black hole-accretion disk system to the line of sight. As a result, blazars, AGN that are viewed down the axis of a radio jet, show significantly different SEDs and variability properties than all other AGN (Bach et al. 2007). Finally, obscuration along the line-of-sight is another key parameter in determining the observational characteristics of an AGN. The vast majority of local optically-selected AGN show evidence for being obscured (e.g., Maiolino \& Rieke 1995; Ho et al. 1997), and these are called Type 2 AGN, while the unobscured sources are defined as Type 1 AGN. Observationally, the obscuration is revealed as significant soft X-ray absorption (e.g., Tozzi et al. 2006), the disappearance of the optical broad permitted lines (Khachikian \& Weedman 1974), and a reddened continuum (Wilkes et al. 2005).

Despite its near ubiquity, the origin of the obscuration around AGN is still unclear. Infrared emission from dust near the sublimation temperature (e.g., Rieke 1978; Neugebauer et al. 1979; Barvainis 1987; Sanders et al. 1989) and variability in the X-ray absorbing column density (Risaliti et al. 2002, 2005) both point to a location $\sim 1 \mathrm{pc}$ from the central engine, although it is difficult to rule out contributions from the host galaxy at larger scales (Maiolino \& Rieke 1995; Ballantyne et al. 2003; Rigby et al. 2006). It is also possible that the origin of the obscuration may arise through a variety of processes for AGN with different luminosities or at different points in their evolution (Ballantyne et al. 2006b).

Perhaps the most significant challenge facing the pc-scale absorber (or obscuring 'torus' in the AGN unification model; Antonucci 1993) is to account for the observed 4-to-1 Type 2/Type 1 ratio of AGN required to fit the hard X-ray background (Gilli et al. 2007). This fact requires the obscuring material to cover $\sim 80 \%$ of the sky as observed from the central black hole, and thus have a structure so that its vertical scale height is of the same magnitude as its radius, i.e. $H / r \sim 1$. Krolik (2007) and Shi \& Krolik (2008) have argued that the AGN emission absorbed and re-radiated in the infrared by dust in the torus can provide enough pressure support to inflate a structure to the required scale. Similarly, Chang et al. (2007) suggested that X-ray heating of the outer accretion disk may be sufficient to result in a $H / r \sim 1$ geometry. Another explanation, and the one considered here in more detail, is feedback from a nuclear starburst disk (Fabian et al. 1998; Wada \& Norman 2002; Thompson et al. 2005).

There are many reasons to consider starburst disks as a source of obscuration in AGN. At the most basic level, gas to fuel the black hole must be funneled toward the center of the galaxy and is therefore likely to cause star 
formation en route. In fact, recent adaptive optics observations by Davies et al. (2007) have shown the presence of a post-starburst population at parsec scales in 9 local Seyfert galaxies, indicating that this may indeed be a common phenomenon. In addition, recent models of AGN tori have suggested that the obscuring region may be clumpy (Nenkova et al. 2002; Dullemond \& van Bemmel 2005; Hönig et al. 2006), a common trait of star-forming regions in starburst galaxies (Förster Schreiber et al. 2001; Snijders et al. 2007). Type 2 AGN also seem to be more correlated with star-forming activity in general than the unobscured Type 1 objects (Cid Fernandes \& Terlevich 1995; González Delgado et al. 2001; Levenson et al. 2001; Kim et al. 2006; Lacy et al. 2007). There is also evidence for a correlation between the observed intensity of star-formation in the host galaxy and the AGN nuclear luminosity (Shi et al. 2007; Watabe et al. 2008).

A more profound aspect of star-formation as a source of AGN obscuration is that it provides a connection between the black hole-accretion disk environment and the host galaxy (e.g., Kawakatu \& Wada 2008). It is now accepted that the correlations between the black hole mass and bulge properties in early-type galaxies (Maggorian et al. 1998; Ferrarese \& Merritt 2000; Gebhardt et al. 2000; Tremaine et al. 2002) are evidence for a significant connection between the growth of the black hole and the build up of a galactic bulge (e.g., Silk \& Rees 1998; Fabian 1999; Kaufmann \& Haehnelt 2000; Wyithe \& Loeb 2003; Murray et al. 2005; Di Matteo et al. 2005; Fabian et al. 2008). Therefore, it is expected that as the black hole and galaxy bulge are growing, there will be significant accretion on to the black hole that is obscured by star formation in the host galaxy (e.g., Hopkins et al. 2005). Indeed, the hard Xray background, emitted by accreting black holes throughout the history of the Universe, has a very hard spectrum, indicative of being dominated by obscured AGN (Setti \& Woltjer 1989; De Luca \& Molendi 2004; Gilli et al. 2007). Interestingly, the redshift distribution of the obscured AGN that dominate the X-ray background peaks at $z \sim 1$, very similar to the peak in the cosmic star-formation history (Tozzi et al. 2001; Barger et al. 2002; Hopkins 2004; Barger et al. 2005). This result may imply a connection between the obscuring material and the evolution of the host galaxy (Fabian et al. 1998; Franceschini et al. 1999; Ballantyne et al. 2006a). In fact, there is tentative evidence that the fraction of obscured AGN does increase with $z$ (Ballantyne et al. 2006a; Treister \& Urry 2006). Thus, if star-forming disks do provide a significant amount of AGN obscuration at $z \sim 1$ (where the X-ray background sources are most common) then by studying the properties and evolution of the absorbing material, we can directly probe the evolution of the underlying host galaxy.

In this paper, we make use of the analytic starburst disk models developed by Thompson et al. (2005) to investigate the properties of starburst disks as a source of obscuring material in the Type 2 AGN found at $z \sim 1$. In the paper by Thompson et al. (2005), the models were able to successfully describe many of the observed proprieties of Ultra-Luminous Infrared Galaxies (ULIRGs), and it was noted that under certain conditions, the photosphere of the starburst disks may reach $H / r \sim 1$. Here, we adapt the model to consider the much less intense star-forming disks that might be expected to be found around the Seyfert-like luminosity AGN that dominate the population at these redshifts (Ueda et al. 2003; Barger et al. 2005). We search for the range of phys- ical parameters these starburst disks must have in order for them to provide significant obscuration. Both radio and far-IR fluxes are predicted to determine if the disks can be (or have already been) detected by current observational surveys. The next section provides a brief review of the Thompson et al. (2005) models, and then describes how the theory was altered and applied to this problem. Section 3 presents the results of our calculations, and describes both the physical and observational properties of starburst disks that may obscure an average AGN. The results are discussed in Section 4 and we present our conclusions in Section 5. When necessary, the following $\Lambda$-dominated cosmology is assumed in this paper: $H_{0}=70 \mathrm{~km} \mathrm{~s}^{-1} \mathrm{Mpc}^{-1}, \Omega_{\Lambda}=0.7$, and $\Omega_{m}=0.3$ (Spergel et al. 2003).

\section{MODELS OF NUCLEAR STARBURST DISKS}

\subsection{Review of Starburst Disk Models}

The properties of the nuclear starburst disks are calculated using the one-dimensional analytic theory developed by Thompson et al. (2005), and we solve the equations presented in their Appendix C. Here, we will briefly review the main assumptions and ideas behind the theory, and refer readers to the paper by Thompson et al. (2005) for a complete discussion.

The starburst disks are calculated as a single phase medium at a radius $r$ from the center of a galaxy with a velocity dispersion $\sigma$ and a supermassive black hole with mass $M_{\mathrm{BH}}$. Further, the disk is assumed to be rotating at the Keplerian rate at all radii, and the gravitational potential is the sum of the pointmass of the black hole and an isothermal sphere describing the galactic bulge. The star-formation in the disk is a local phenomenon and is assumed to always adjust so that Toomre's $Q$ parameter is unity; that is, $Q=\kappa_{\Omega} c_{s} / \pi G \Sigma_{\mathrm{g}}=1$, where $\kappa_{\Omega}$ is the epicycle frequency, $\Omega$ is the Keplerian frequency, $c_{s}$ is the sound speed and $\Sigma_{\mathrm{g}}$ is the gas surface density. This condition on $Q$ allows the gas density $\rho$ at any radius to be immediately written as a function of $\Omega(r)$. The vertical support of the disk is the infrared (IR) radiation pressure of the starburst against dust (the UV optical depth is always much greater than unity in these disks, so the vast majority of the radiation is re-radiated in the mid-and far-IR). Therefore, these starbursts are locally radiating at their Eddington limit. The vertical optical depth of the disk in the IR is $\tau=\Sigma_{\mathrm{g}} \kappa / 2$, where $\kappa(T, \rho)$ is the Rosseland mean opacity.

Fuel is fed to the outer radius of the disk at a rate of $\dot{M}_{\text {out }}$. An unspecified global torque acts on the disk allowing the removal of angular momentum and gas to accrete slowly inwards at a rate $\dot{M}$. This torque is assumed to allow the radial velocity of the gas to be equal to a constant fraction $m$ of the local sound speed; thus, $\dot{M}=2 \pi r \Sigma_{g} m c_{s}$. However, gas can be removed from the disk by local star-formation, so that the accretion rate through the disk can also be written as $\dot{M}=\dot{M}_{\text {out }}-\int_{r}^{R_{\text {out }}} 2 \pi r^{\prime} \dot{\Sigma}_{*} d r^{\prime}$, where $\dot{\Sigma}_{*}$ is the starformation rate (SFR) per unit area. Therefore, there is a competition between star-formation using up the gas in the disk and the torque attempting to transport material through the disk. As discussed in Thompson et al. (2005), this can be understood by considering the advection timescale through the disk, $\tau_{\mathrm{adv}}=r / v_{r}$, and the star-formation timescale, $\tau_{*}=1 /(\eta \Omega)$, where $v_{r}$ is the radial velocity of the gas, and $\eta$ is the star-formation efficiency. If $\tau_{\text {adv }}<\tau_{*}$ then material can move through the disk quickly enough that it can fuel a black hole while forming stars. Thompson et al. (2005) showed that 
by equating these timescales at the outer radius $R_{\text {out }}$, one derives a critical accretion rate, $\dot{M}_{\text {crit }}$, below which nearly all the gas is converted into stars at large radii.

The model disks are calculated from $R_{\text {out }}$ inwards until a point is reached where dissipation by disk accretion dominates the heating over star-formation. At this point, $Q=1$ can no longer be maintained and the starburst disk structure calculation is terminated. The actual value of the inner radius depends on the particular parameters of the model, but is typically $<1$ pc.

\subsection{A Survey of Starbursts}

Thompson et al. (2005) calculated starburst disk structures appropriate for ULIRGs, but the X-ray background is dominated by AGN whose host galaxies must have, on average, SFRs much lower than ULIRGs (Ballantyne \& Papovich 2007). Thus, we have to calculate new starburst models for this different scenario. Since it is clear that it is possible for starburst disks to potentially obscure an AGN (Thompson et al. 2005), these new models are designed to cover a wide range of parameter space to determine the variety of properties that AGN-obscuring starburst disks may have.

Each starburst disk calculation has 5 input parameters that were varied through different values: $\log \left(M_{\mathrm{BH}} / M_{\odot}\right)=7$, $7.5,8,8.5$ (the galactic velocity dispersion is then calculated through the $M_{\mathrm{BH}}-\sigma$ relation: $M_{\mathrm{BH}}=2 \times 10^{8} \sigma^{4}$; e.g., Tremaine et al. 2002); $m=0.0075,0.01,0.025,0.05,0.075$, $0.1,0.2 ; R_{\text {out }}=50,100,150,200,250 \mathrm{pc} ; f_{\text {gas }}=0.1$, $0.5,0.9$ (the gas fraction determines $\dot{M}_{\text {out }}$ and decreases to smaller $r$; Thompson et al. 2005); and the dust-to-gas ratio of the gas (either $1 \times, 5 \times$ or $10 \times$ the local ISM value). As emphasized by Thompson et al. (2005), the starburst disk structure is very sensitive to the temperature dependence of the Rosseland mean opacity. Similarly, the overall opacity value is an important parameter, as increasing the opacity can result in a larger temperature without necessarily enhancing the SFR. There are numerous observational hints that the region around an AGN may have enhanced metallicity (Hamann \& Ferland 1999; Nagao et al. 2006; Arav et al. 2007), as well as increased grain growth (Chang et al. 2007 and references therein). Therefore, it is important to calculate starburst disks with differing dust-to-gas ratios. Following Thompson et al. (2005), we use the Rosseland mean opacity curve calculated by Semenov et al. (2003) with the following parameters: a density of $10^{6} \mathrm{~cm}^{-3}$, "normal" silicates, homogeneous distribution of dust materials, and spherical particles. To determine the opacity at different dust-to-gas ratios, this basic curve, appropriate for typical ISM values, was multiplied by the desired ratio (Ferguson et al. 2007).

Starburst disk models were run for each permutation of the input parameters resulting in a total of 1260 distinct starburst disk structures. However, before analyzing the properties of these disk models, we must select the models that are most likely to both fuel and obscure a central active nucleus (i.e., ones where $\tau_{\mathrm{adv}}<\tau_{*}$ ). The potential for obscuring an AGN arises when the central temperature of the starburst disk surpasses the sublimation temperature of the dust (Thompson et al. 2005). At that point, the opacity of the gas drops precipitously and the SFR rises dramatically in order to keep $Q=1$. In this situation, the effective temperature of the disk (roughly the surface temperature) may remain below the sublimation temperature, leading to a significant vertical

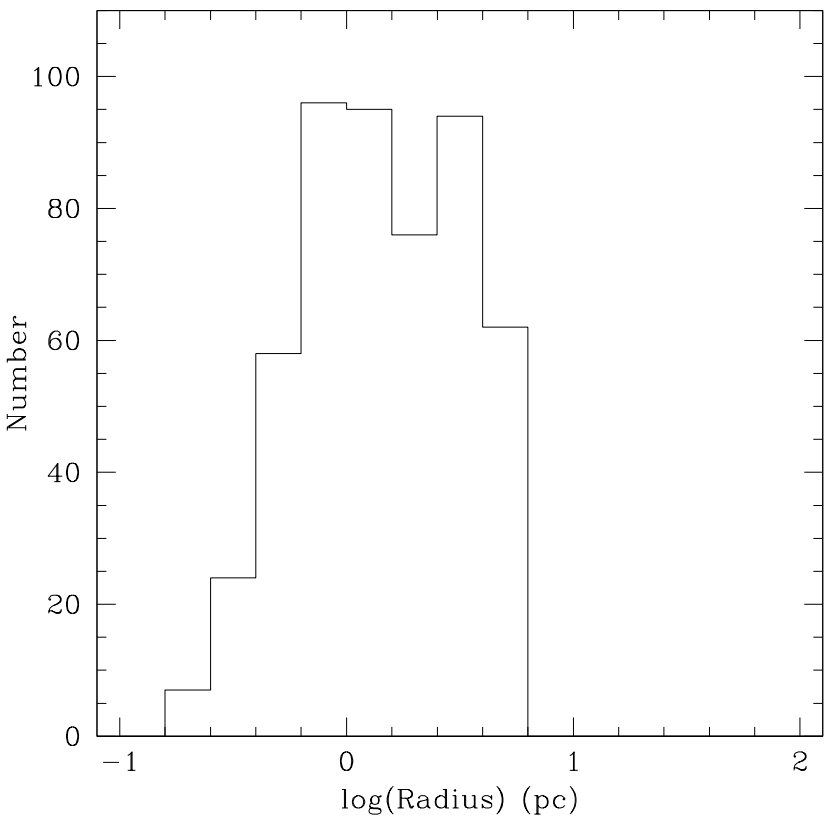

FIG. 1.- Histogram of the radius where the SFR reaches its maximum The models making up the histogram were selected so that the maximum star-formation rate occurred in the high temperature dust sublimation region that satisfied the two criteria described in the text. Of the 1260 starburst disk models calculated, a total of 512 , or $41 \%$, satisfy these conditions. The peak star-formation rate always occurs at $r \gtrsim 0.2 \mathrm{pc}$, with $r \approx 1$ and $3 \mathrm{pc}$ being the two most common values.

opacity gradient in the disk. Thompson et al. (2005) argued that, although the disk may remain thin with a $H / r<1$, the photosphere of the disk could puff up to $H / r \sim 1$. Therefore, each of the 1260 models was searched to determine if there existed a region that satisfied both of the following conditions:

1. the central temperature was greater than $900 \mathrm{~K}$,

2. the SFR was greater than $10 \%$ of the SFR at $R_{\text {out }}$.

The second criterion was necessary to exclude a small number of models that marginally exceeded the temperature condition, but did not reach the sublimation temperature. Only 512 models, or $41 \%$ of the calculations, produced an inner starburst that satisfied both of the imposed conditions. A histogram of the radius at which the SFR reached its maximum within this region is shown in Figure 1. The histogram shows that the most common radius to find this burst of starformation is $r \approx 1-3 \mathrm{pc}$. Such radii are very similar to the scales of the obscuring medium measured in nearby AGN (Jaffe et al. 2004; Tristram et al. 2007). Thus, these starburst disk models reproduce the size scales necessary to obscure AGN. It is worth emphasizing that in choosing the range of model parameters, we made no a priori assumptions about what values would be more (or less) likely to produce these pc-scale starbursts, but only considered values to be typical of observed Seyfert-like galaxies. The distributions of observables shown below are derived solely from the models shown in Fig. 1, which we consider to be those that are most likely to obscure the central AGN.

\section{RESULTS}

\subsection{Physical Properties of the Starburst Disks}




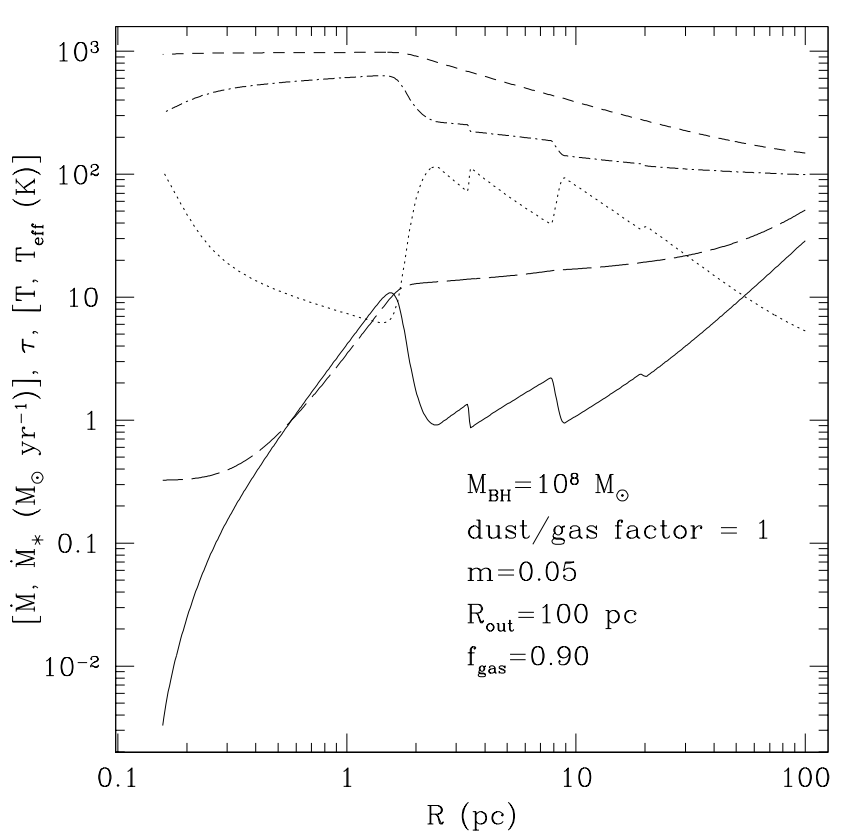

FIG. 2.- Plot of the mass accretion rate $\dot{M}$ (long-dashed line; in $\left.\mathrm{M}_{\odot} \mathrm{yr}^{-1}\right)$, the SFR $\dot{M}_{*}\left(\right.$ solid line; in $\left.\mathrm{M}_{\odot} \mathrm{yr}^{-1}\right)$, the vertical optical depth $\tau$ (dotted line), the central temperature $T$ (short-dashed line; in $\mathrm{K}$ ), and the effective temperature $T_{\text {eff }}$ (dot-dashed line; in $\mathrm{K}$ ), all as a function of radius. The data are drawn from a starburst disk model with the following parameters: $M_{\mathrm{BH}}=10^{8} \mathrm{M}_{\odot}$, dust-to-gas factor $=1, m=0.05, R_{\mathrm{out}}=100 \mathrm{pc}$ and $f_{\text {gas }}=0.9$.

As a guide to the physical structure of one of the starburst disks, Figure 2 plots the radial dependence of several interesting variables from one representative model. The details behind the models are described by Thompson et al. (2005), but it is worth emphasizing here that star-formation occurs throughout the disk (solid line), from $R_{\text {out }}=100 \mathrm{pc}$ to the inner-radius at $\lesssim 1 \mathrm{pc}$. However, the central temperature of the disk (short-dashed line) increases to smaller radii as the rising density causes the disk to become more optically-thick to the stellar radiation. Eventually, this temperature reaches the sublimation temperature of the dust, and the opacity drops (dotted line), resulting in a significant burst of star-formation. Since the effective temperature (dot-dashed line) is still below this sublimation temperature, a significant vertical opacity gradient will exist in this region. Thus, this is the key region for where star-formation can puff up the disk photosphere to $H / r \sim 1$, and not at larger radii, even if the SFRs are comparable. This inner burst of star-formation nearly exhausts the gas supply in these models, but a small amount survives to make its way onto the inner accretion disk to feed the black hole (long-dashed line).

We now turn to the physical properties for the ensemble of 'successful' models shown in Fig. 11 What is necessary for these disks to be able to obscure an AGN on pc scales? The solid lines in Figure 3 show the distributions of the input parameters derived from those models that have a pc-scale burst of star-formation. These plots show that successful models can be produced at every value of the input parameters, but pc-scale starbursts are more commonly generated if the disks have specific properties. For example, panel (a) shows that more massive black holes can more easily produce such starbursts. This is perhaps unsurprising since in these $Q=1$ structures a larger black hole mass results in a denser and hence hotter starburst disk (Thompson et al. 2005). In contrast, panel (b) shows that a smaller starburst disk is more likely to have the inner ring of star-formation. Smaller starburst disks retain a larger fraction of their gas at a given radius than larger disks. Thus, the opacity is greater and the temperature can more easily reach the sublimation temperature of dust. The smallest $R_{\text {out }}$ considered, $50 \mathrm{pc}$, is an interesting number as its roughly the same size as the star-forming regions found around nearby Seyferts by Davies et al. (2007). The models considered here do show that such small starburst disks are more likely to produce an AGN obscuring region than larger disks, in agreement with the observations.

There is a similarly strong trend shown in panel (c) involving the Mach number $m$. A larger value of $m$ reduces $\tau_{\mathrm{adv}}$, and thus causes more efficient mass accretion through the starburst disk. Figure 3 shows that an inner burst of star formation at pc-scales is more common at higher Mach numbers. In fact, $\sim 50 \%$ of such models had $m \geq 0.1$. As with the smaller values of $R_{\text {out }}$, efficient mass accretion results in a larger fraction of gas at pc-scales, which increases the opacity and hence temperature of the gas. The same reasoning can also be applied to the dust-to-gas factor shown in panel (d), where clearly a larger dust-to-gas ratio results in a more common inner pc-scale burst of star-formation. A enhanced dust-to-gas ratio increases the vertical opacity to the starburst radiation, leading to larger temperatures and thus increasing the likelihood of surpassing the sublimation temperature.

The gas fraction at $R_{\text {out }}$ (Fig. 3 (e)) is another important parameter for the existence of a pc-scale starburst, with $f_{\text {gas }}=$ 0.1 producing only $\sim 2 \%$ of the 'successful' models. The mass accretion rate at $R_{\text {out }}$ is directly related to $f_{\text {gas }}$, so that a larger accretion rate onto the outer disk is the result of increasing $f_{\text {gas }}$. Hence, it is easier to exceed the $\dot{M}_{\text {crit }}$ crucial for feeding and obscuring the black hole. A larger $f_{\text {gas }}$ at $R_{\text {out }}$ will also likely result in a larger fraction of gas on pc-scales, and the extra heating argument described above will hold. The accretion rate varies as $f_{\text {gas }}^{2}$ (Thompson et al. 2005), thus models with $f_{\text {gas }} \sim 0.5$ are adequate to produce potentially AGN-obscuring starburst disks.

It is clear from the physics of the starburst model that in order to produce this pc-scale burst of star-formation that may obscure an AGN, the temperature of the disk must exceed the sublimation temperature of dust producing a sudden drop of the vertical opacity. Figure 3 shows that this situation is more likely to occur if one of the following is true: a highmass black hole, a smaller starburst disk, efficient angular momentum transport in the disk, a reasonable gas mass fraction, or a large dust-to-gas ratio. However, not all of these conditions have to be simultaneously satisfied. For example, the dotted lines in Fig. 3 show the distribution of $R_{\text {out }}$, $f_{\text {gas }}$ and the dust-to-gas factor for the subset of models where $\log \left(M_{\mathrm{BH}} / M_{\odot}\right)=7$ and $m=0.025$, two non-ideal conditions for the existence of the inner pc-scale starburst. In this case, the small starburst disks and large dust-to-gas ratios are basically required in order to generate a potentially AGN-obscuring starburst. Therefore, while it may be harder to produce a pc-scale burst of star-formation around a smaller mass black hole, it is not impossible if the other properties of the starburst disk are favorable.

\subsection{Star-formation Rates}



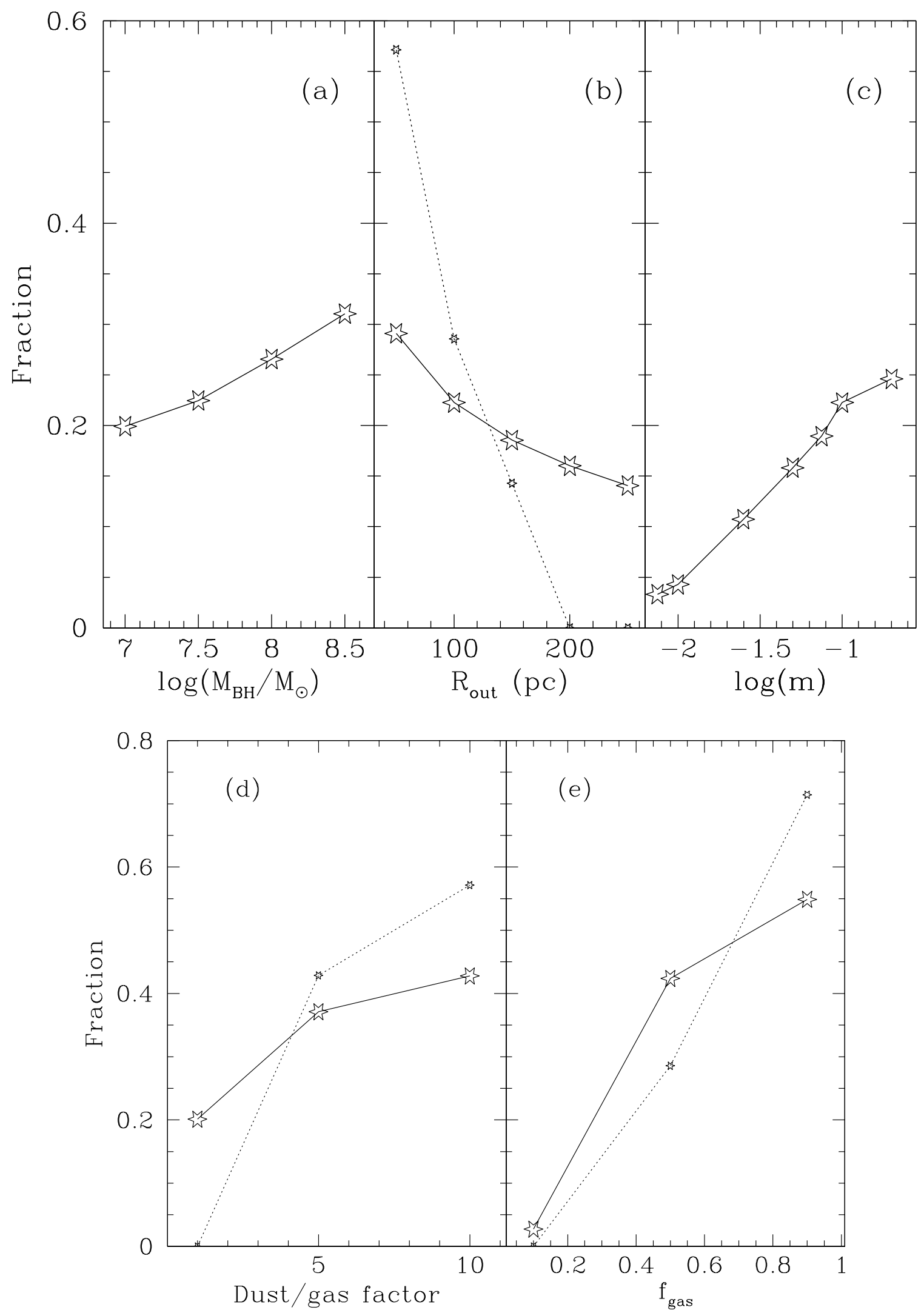

FIG. 3.- The different panels show the fraction of the models with pc-scale bursts of star-formation (i.e., those shown in Fig. 1 that have a specific input parameter. The panels show (a) the mass of the central black hole, (b) the outer radius of the starburst disk, (c) the logarithm of the Mach number $m$, (d) the dust-to-gas multiplicative factor and (e) the gas fraction at $R_{\text {out }}$. The solid lines are the results when including all the successful models. The dotted lines plot the fractions when the additional constraints of $\log \left(M_{\mathrm{BH}} / M_{\odot}\right)=7$ and $m=0.025$ are imposed. 


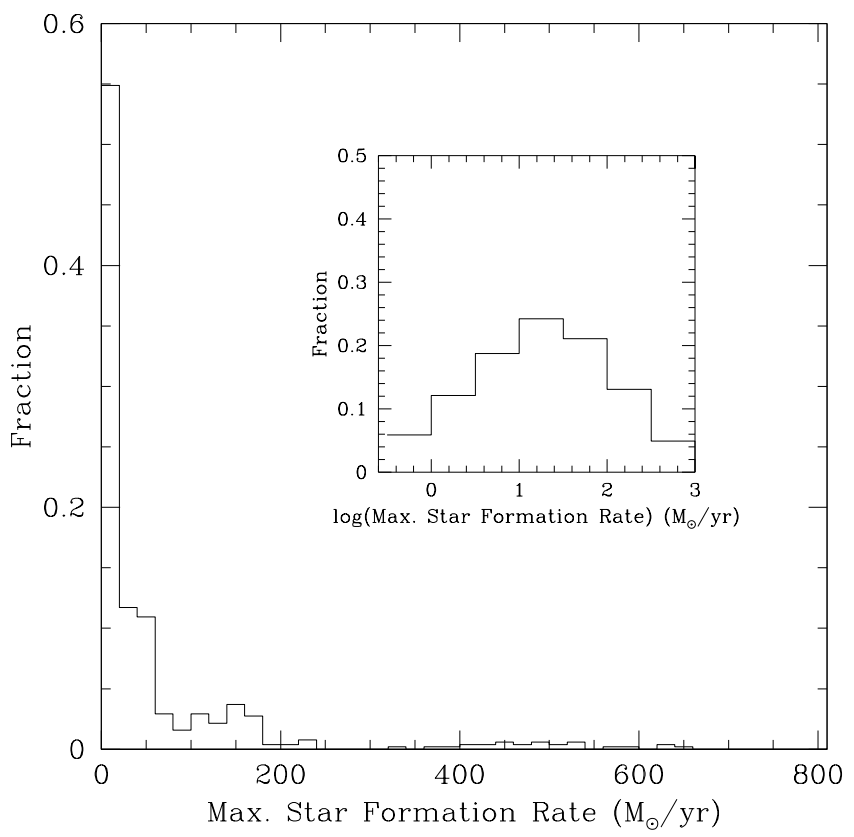

FIG. 4.- Histogram of the maximum SFRs found in the models with pc-scale starbursts. About 55\% of the models have a maximum SFR $<$ $20 \mathrm{M}_{\odot} \mathrm{yr}^{-1}$. The distribution shows a weak tail to high SFRs, with a small number of models with peak SFRs $>300 \mathrm{M}_{\odot} \mathrm{yr}^{-1}$. The inset shows the same distribution, but now on a logarithmic axis. The most common SFRs are $10-30 \mathrm{M}_{\odot} \mathrm{yr}^{-1}$ and only $\sim 5 \%$ of the 'successful' calculations have SFRs $>300 \mathrm{M}_{\odot} \mathrm{yr}^{-1}$.

We now understand the conditions necessary for the starburst disk to produce a potentially AGN obscuring ring of star-formation. Focusing in on the star-formation events themselves, Figure 4 plots the distribution of maximum SFRs found in these pc-scale starbursts. The plot shows that just over half the models have maximum SFRs $<20 \mathrm{M}_{\odot} \mathrm{yr}^{-1}$ (with values $\sim 10 \mathrm{M}_{\odot} \mathrm{yr}^{-1}$ most common), rates that are very typical of luminous IR galaxies (LIRGs; Wang et al. 2006), but much less than those observed in ULIRGs ( $100 \mathrm{M}_{\odot} \mathrm{yr}^{-1}$; Rodríguez Zaurín et al. 2008). However, the star-formation per unit area in the model bursts are very high $\left(\sim 10^{6} \mathrm{M}_{\odot} \mathrm{yr}^{-1} \mathrm{kpc}^{-2}\right)$, exceeding those observed in ULIRGs $\left(\sim 10^{3} \mathrm{M}_{\odot} \mathrm{yr}^{-1} \mathrm{kpc}^{-2}\right.$; Graciá-Carpio et al. 2008). Thus, these pc-scale starbursts are basically miniature ULIRGs: small regions of very efficient star-formation, but because the area is so small, the maximum SFRs can be LIRG-like. Interestingly, Ballantyne \& Papovich (2007) showed that the host galaxies of the AGN that dominate the Xray background must, on average, have SFRs $<100 \mathrm{M}_{\odot} \mathrm{yr}^{-1}$ to avoid violating the cosmic IR background. This constraint is consistent with the predictions of Fig. 4, where $\sim 80 \%$ of the models have maximum SFRs less than $100 \mathrm{M}_{\odot} \mathrm{yr}^{-1}$.

The distribution of SFRs shows a small number of models with maximum SFRs $>300 \mathrm{M}_{\odot} \mathrm{yr}^{-1}$. The Fig. 4 inset indicates that only $5 \%$ of the models present such ULIRGlike SFRs. These massively star-forming disks only occur when $\log \left(M / M_{\odot}\right)=8.5, m=0.1$ or 0.2 , and $f_{\text {gas }}>0.90$. Clearly, significant gas resources are necessary to power such bursts of star-formation.

\subsection{Observational Consequences 3.3.1. AGN Properties}

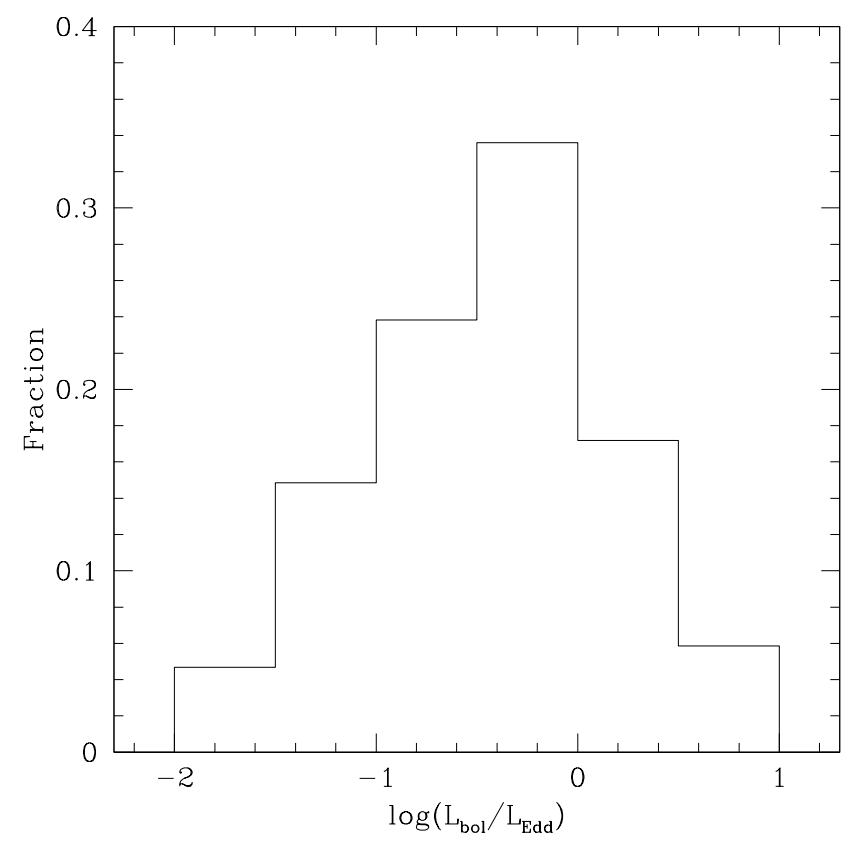

FIG. 5.- Histogram of the Eddington ratio, or $L_{\mathrm{bol}} / L_{\mathrm{Edd}}$, predicted from a central AGN being fed from the surrounding starburst. Mass-loss in the inner accretion disk is not included, so these ratios are strict upper-limits. Sixty percent of the models have $0.1 \leq L_{\mathrm{bol}} / L_{\mathrm{Edd}} \leq 1$, with a further $\sim 20 \%$ apparently accreting at super-Eddington rates.

As mentioned in Sect. 3.1 if $\tau_{\mathrm{adv}}<\tau_{*}$ a small amount of gas survives its passage through the starburst disk and will feed onto the sub-pc accretion disk, and may ultimately accrete onto the black hole. Under the assumption that the accretion rate at the inner-most edge of the starburst disk is the same as the accretion onto the black hole, the bolometric luminosity of the resulting AGN can be estimated from $L_{\mathrm{bol}}=\eta \dot{M} c^{2}$, where $\eta=0.1$ is the radiative efficiency of the accretion disk. This is not a very realistic assumption, since outflows from the central regions of AGN are commonly observed, as well as being a standard prediction from numerical simulations of accretion flows (e.g., Crenshaw et al. 2003; Hawley \& Krolik 2006). However, these outflows ensure that the accretion rate onto the black hole is always less than the accretion rate at the outer edge of the accretion disk, thus the luminosities calculated from the predicted $\dot{M}$ are strict upperlimits ${ }^{1}$. A further, implicit, assumption in this calculation is that there are no other sources of fuel for the black hole aside from that provided by the starburst disk.

Keeping those assumptions in mind, we plot in Figure 5 the Eddington ratio $L_{\mathrm{bol}} / L_{\mathrm{Edd}}$ distribution from the models with pc-scale starbursts. Here, $L_{\mathrm{Edd}}=4 \pi G M_{\mathrm{BH}} m_{p} c / \sigma_{\mathrm{T}}$ is the Eddington ratio for spherical accretion, where $m_{p}$ is the proton mass and $\sigma_{\mathrm{T}}$ is the Thomson cross-section. The histogram shows a log-normal-like distribution with a peak at $L_{\mathrm{bol}} / L_{\mathrm{Edd}} \approx 0.5$. In all, about $20 \%$ of the models have Eddington ratios less than $0.1, \sim 60 \%$ have ratios $0.1 \leq$ $L_{\mathrm{bol}} / L_{\mathrm{Edd}} \leq 1$, while $\sim 20 \%$ predict super-Eddington accre-

\footnotetext{
${ }^{1}$ The mass-loss rate from an accretion disk depends on the location from where it is launched and how it is driven, both extremely uncertain numbers for almost all AGN (Crenshaw et al. 2003). Recently, Miller et al. (2008) argued that an outflow from the Galactic black hole GRO J1655-40 was launched from the disk by magnetic processes. The mass-loss rate in this case was estimated to be $>5-10 \%$ of the accretion rate.
} 


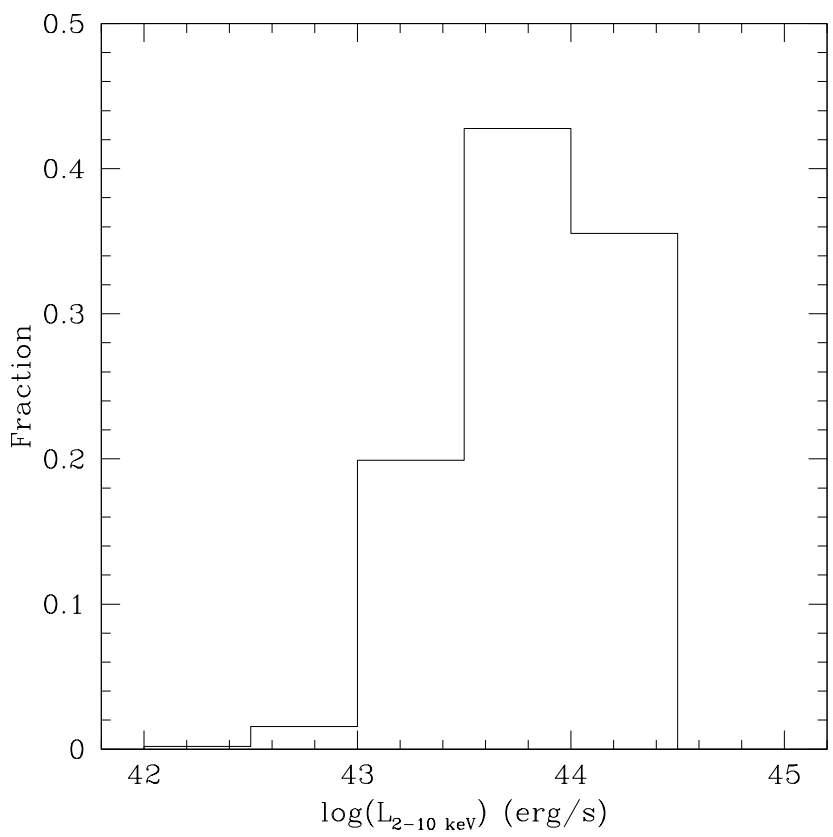

FIG. 6.- Histogram of the 2-10 keV luminosity of AGN fed from the starbursts disks with pc-scale bursts of star-formation. These are strict upperlimits. The range of luminosities is consistent with those AGN observed to dominate the X-ray background at $z \sim 1$ (Ueda et al. 2003; Barger et al. 2005).

tion. Since these ratios will be upper-limits, we find that the majority of the Eddington ratios predicted by fueling from the model starburst disks are consistent with the values inferred from obscured, $z \sim 1$ AGN (e.g. Ballo et al. 2007).

While the Eddington ratio is an interesting parameter, it is not a true observable. Assuming a standard radiativelyefficient AGN spectrum, bolometric corrections can be used to determine the AGN luminosity in the $2-10 \mathrm{keV}$ X-ray band. Recently, Vasudevan \& Fabian (2007) determined the 2-10 keV bolometric correction for a number of nearby AGN with both X-ray and UV data. These authors found that the bolometric correction may depend on the Eddington ratio of the AGN, with a value of $L_{\mathrm{bol}} / L_{X} \sim 20$ for $L_{\mathrm{bol}} / L_{\mathrm{Edd}}<$ 0.1 , while $L_{\mathrm{bol}} / L_{X} \sim 55$ for larger values, where $L_{X}$ is the AGN luminosity in the $2-10 \mathrm{keV}$ band. Employing these bolometric corrections, strict upper-limits to $L_{X}$ can be calculated for our models and its distribution is plotted in Figure 6 . Over $90 \%$ of the predicted X-ray luminosities fall between $L_{X}=10^{43} \mathrm{erg} \mathrm{s}^{-1}$ and $10^{44.5} \mathrm{erg} \mathrm{s}^{-1}$, which is precisely the range of luminosities observed from the AGN that dominate the hard X-ray background at $z \sim 1$ (Ueda et al. 2003; Barger et al. 2005). It is interesting that although the nuclear accretion process is not modeled, the AGN accretion rates predicted by the competition between star-formation and a global feeding process on scales of tens of pc gives such good agreement with the observations. Although the details are unclear, this result may support models of AGN feeding through star-forming disks and global, bar-like instabilities (see Sect.4.3).

\subsubsection{The Starburst Disk}

To determine the observational properties of the starburst disk itself, we calculate a simple SED for each model assuming blackbody emission at each radius and a face-on viewing

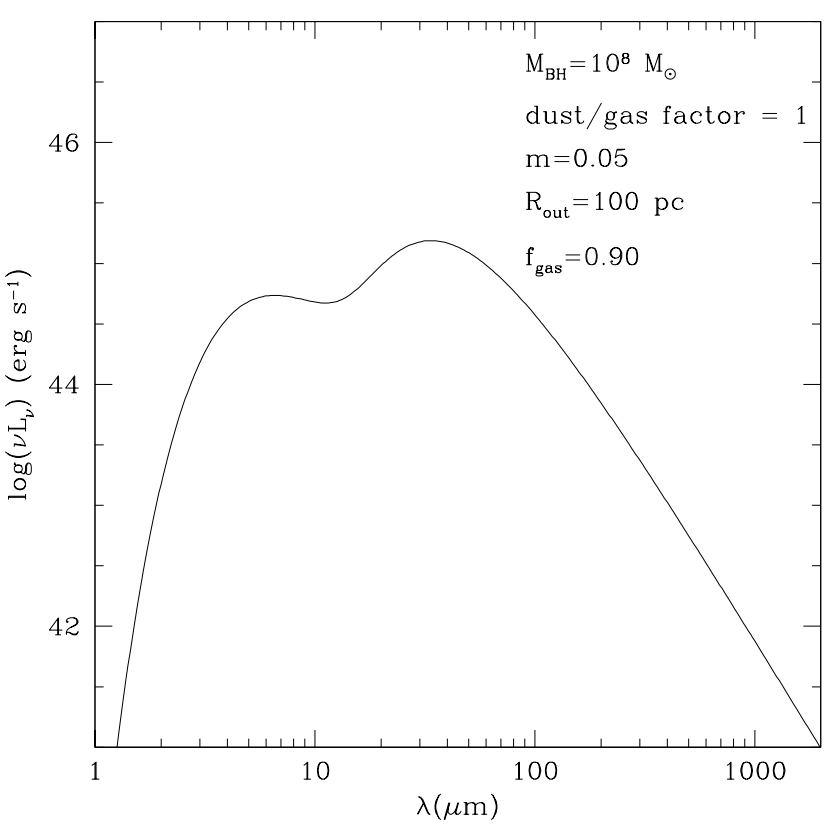

FIG. 7. - Example SED of a starburst disk from a model with the following parameters: $M_{\mathrm{BH}}=10^{8} \mathrm{M}_{\odot}$, dust-to-gas factor $=1, m=0.05$, $R_{\text {out }}=100 \mathrm{pc}$ and $f_{\text {gas }}=0.9$. The inner pc-scale starburst causes a bump in emission at $\lambda \sim 5 \mu \mathrm{m}$. The SED is calculated from eq. 1 and assumes a face-on geometry; hence the luminosity is an upper-limit.

angle (Thompson et al. 2005):

$$
\lambda L_{\lambda}=\frac{2 \pi h c^{2}}{\lambda^{4}} \int_{R_{\text {in }}}^{R_{\text {out }}} \frac{2 \pi r d r}{\exp \left[h c / \lambda k_{\mathrm{B}} T_{\mathrm{eff}}(r)\right]-1},
$$

where $R_{\text {in }}$ is the inner radius for each model, $h$ is Planck's constant and $k_{\mathrm{B}}$ is Boltzmann's constant. An example SED, calculated from the same starburst model as described in Fig. 2, is shown in Figure 7. The SED shows two maxima: one at $\sim 30 \mu \mathrm{m}$ from the star-formation at the outer edge of the disk, and one at $\sim 5 \mu \mathrm{m}$ from the pc-scale starburst. This shorter-wavelength peak is a generic feature of the models with the inner ring of star-formation, and is due to the loss of dust opacity in this region that limits the reprocessing available for the hot starburst emission. Thus, the effective temperature at these radii moves into the near-IR regime. As these SEDs are calculated assuming the disk is viewed face-on, the luminosities and fluxes discussed below are upper-limits.

Figure 8 plots the distribution of $24 \mu \mathrm{m}$ flux at $z=0.8$ predicted from the starburst disk SEDs. The choice of $z=0.8$ is illustrative, but is a common redshift for the population of obscured AGN that we wish to describe (e.g., Barger et al. 2005). The most common $24 \mu \mathrm{m}$ flux predicted by the starburst models is $\sim 1 \mathrm{mJy}$, but values can be as low as $10 \mu \mathrm{Jy}$, or as high as $30 \mathrm{mJy}$. As mentioned above, these values should be treated as upper-limits. The blue shaded region shows where the models with a maximum $\mathrm{SFR} \leq 30 \mathrm{M}_{\odot} \mathrm{yr}^{-1}$, while the red area plots models with a maximum SFR> $100 \mathrm{M}_{\odot} \mathrm{yr}^{-1}$. The starburst disks with lower SFRs, make up over $50 \%$ of the models (Fig. (4) and dominate the $24 \mu \mathrm{m}$ predictions at fluxes $<1 \mathrm{mJy}$. For fluxes between 1-3 mJy, the models with intermediate SFRs dominate before ceding to the small number of high SFR disks.

The fluxes shown in Fig. 8 are well within the sensitivity limits of many of the deep Spitzer surveys over the last several 


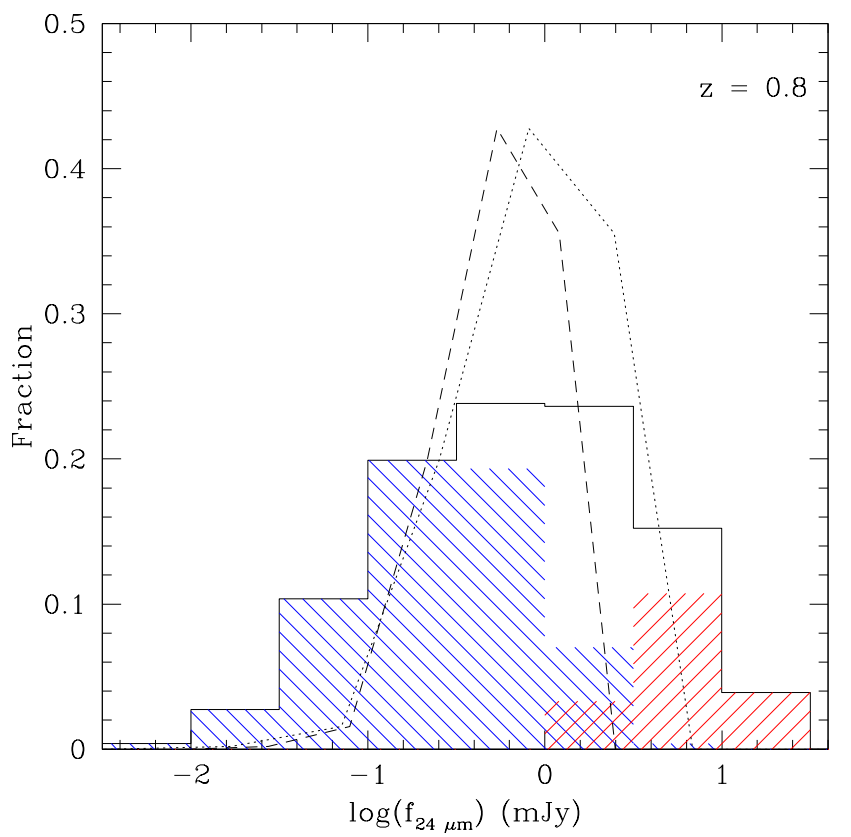

FIG. 8. - The solid line plots the distribution of predicted $24 \mu \mathrm{m}$ flux at $z=$ 0.8 from models with pc-scale starbursts. The blue region denotes the location of models with a maximum $\mathrm{SFR} \leq 30 \mathrm{M}_{\odot} \mathrm{yr}^{-1}$, while the red region shows the distribution of models with a maximum SFR $>100 \mathrm{M}_{\odot} \mathrm{yr}^{-1}$. All of these fluxes are derived from SEDs calculated by equation 1 and are thus upper-limits. The dashed and dotted lines are predictions of the $24 \mu \mathrm{m}$ flux due to AGN heating of a constant density torus at a distance of 1 and $10 \mathrm{pc}$, respectively (Ballantyne et al. 2006b). These calculations made use of the distribution of X-ray luminosities from Fig. 6

years (Lacy et al. 2004; Rigby et al. 2004; Franceschini et al. 2005; Stern et al. 2005; Alonso-Herrero et al. 2006; Barmby et al. 2006; Brand et al. 2006; Pérez-González et al. 2008). Thus, if these disks exist around AGN, they should have been detected by these surveys. However, the AGN itself can heat dust to near- and mid-IR emitting temperatures. The calculations presented in this paper do not include the feedback of the central AGN on the starburst disk. To estimate the $24 \mu \mathrm{m}$ flux from AGN heating, we make use of the calculations by Ballantyne et al. (2006b) who modeled AGN SEDs from the X-ray to the far-IR assuming a constant density torus with no star-formation. These models allow one to easily connect the X-ray luminosity to predicted IR fluxes, so the distribution of X-ray luminosities shown in Fig. 6 can then be converted to a distribution of $24 \mu \mathrm{m}$ flux with the results plotted in Fig. 8 The dashed and dotted lines assume a distance from the AGN to the inner radius of the torus of 1 and $10 \mathrm{pc}$, respectively. The lines were derived from models that are averaged over all X-ray column densities, and assume a $(1+z)^{0.3}$ evolution of the AGN Type 2/Type 1 ratio (Ballantyne et al. 2006a; Treister \& Urry 2006), although this assumption does not affect the results shown here. We see that a dust torus heated by a distribution of AGN X-ray luminosities from Fig. 6results in very similar $24 \mu \mathrm{m}$ fluxes as that predicted from the starburst disks. Thus, we recover the long-standing problem of attempting to determine the relative influence of star-formation and AGN heating in an observed spectrum. However, the nuclear starburst models described here present a new wrinkle: observable hot-dust emission in the near- and mid-IR that is not caused by AGN heating. This may mislead some methods of AGN identification in the IR

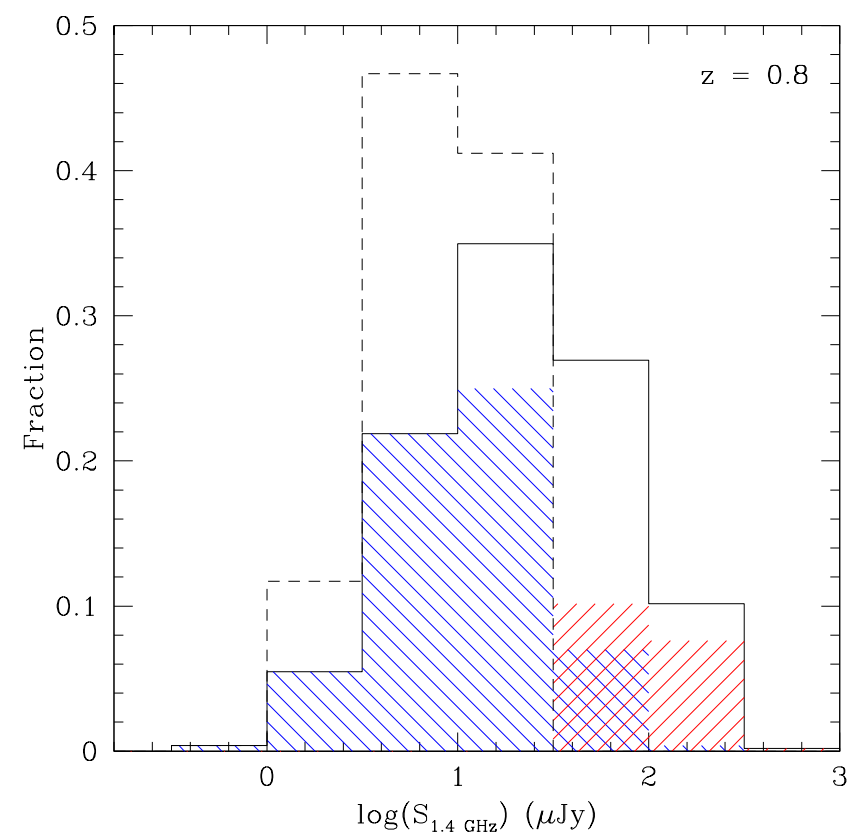

FIG. 9.- The solid histogram plots the distribution of $1.4 \mathrm{GHz}$ radio fluxes at $z=0.8$ as estimated from the radio-IR correlation of Bell (2003). As in Fig. 8 the blue and red regions denote the location of the models with maximum SFRs $<30$ and $>100 \mathrm{M}_{\odot} \mathrm{yr}^{-1}$, respectively. As these radio fluxes were derived from the starburst SEDs, they should also be treated as upperlimits. The dashed histogram is an estimate of the nuclear radio-emission from the central AGN assuming it is radio quiet, has a flat spectrum of $\alpha=0$ and $\nu L_{\nu}(5 \mathrm{GHz})=10^{-5} L_{2-10 \mathrm{keV}}$ (Terashima \& Wilson 2003).

that are based on the shape of the near- and mid-IR spectrum (e.g., Donley et al. 2007). We conclude that the nuclear starburst disks will be easily visible in the mid-IR, but since the predicted fluxes are nearly identical to those expected from AGN heating, it will be extremely difficult to positively identify them or measure their properties (see Sect. 4).

Another observational consequence of the nuclear starburst disks will be radio emission. There have been numerous attempts to link the properties of AGN found in deep radio and X-ray surveys (Bauer et al. 2002; Georgakakis et al. 2004; Simpson et al. 2006; Barger et al.|2007; Richards et al. 2007; Rovilos et al. 2007; (Park et al. 2008), but the matched sources seems to only comprise a small fraction of all AGN (e.g., Barger et al. 2007). Radio fluxes at $1.4 \mathrm{GHz}$ can be predicted for the starburst disk by making use of the IR SEDs and the radio-IR correlation ${ }^{2}$ (e.g., Yun et al. 2001). We specifically use the form of the radio-IR correlation derived by Bell (2003) that employs the total $8-1000 \mu \mathrm{m}$ luminosity to make sure we account for the emission from the pc-scale starburst. Observations by Appleton et al. (2004) and Ibar et al. (2008) have shown that the local radio-IR correlation does seem to hold at high $z$ (but see Beswick et al. 2008). Therefore, upon assuming a typical starburst radio spectrum of $\alpha=0.8$ (Yun et al. 2001, where $S_{\nu} \propto \nu^{-\alpha}$ ), we plot in Figure 9 the distribution of radio fluxes from the 'successful' starburst disks at a representative redshift of $z=0.8$. As in the previous figure showing the $24 \mu \mathrm{m}$ predictions, the contribution from mod-

\footnotetext{
2 The assumption that such intense starbursts will follow the observed radio-IR correlations may be problematic, as this might require very large magnetic energy densities (e.g., Thompson et al.2006). This problem will be investigated in future work.
} 
els with a maximum $\mathrm{SFR} \leq 30$ and $>100 \mathrm{M}_{\odot} \mathrm{yr}^{-1}$ are shown by the blue and red hatched regions, respectively. The radio flux estimates are again upper-limits as they originate from the starburst IR SEDs. The most common radio flux predicted for these starburst disks is $\sim 10-30 \mu \mathrm{Jy}$, values that will again be dominated by disks with maximum SFRs $\leq 30 \mathrm{M}_{\odot} \mathrm{yr}^{-1}$. This is an interesting flux because it is just below the sensitivity limit $(\sim 40-50 \mu \mathrm{Jy})$ of the deepest radio surveys (e.g., Bauer et al. 2002; Barger et al. 2007). Indeed, Bauer et al. (2002) found that the fraction of X-ray AGN with radio matches increased as they approached their sensitivity limit, and suggested that deeper observations will find a higher fraction of matches. This conclusion agrees with the predictions of Fig. 9 .

Interestingly, Georgakakis et al. (2004) and Richards et al. (2007) find a slight tendency for the fraction of radio/X-ray matches to increase with X-ray obscuration. That is, the more obscured an AGN, the more likely that it will have a radio counterpart in the deep radio surveys. This result, although still tentative (Rovilos et al. 2007), is consistent with our proposed scenario where obscured AGN should be associated with significant star-formation. Future work is needed in the modeling to connect the starburst disks to predictions of X-ray column densities. Further deep radio observations are also required to confirm this trend.

As with the $24 \mu \mathrm{m}$ flux, a major observational difficulty is determining whether the origin of the radio flux is due to the AGN or to star-formation. It has long been known that the radio source population seems to change at a flux of $\sim 1 \mathrm{mJy}$, with radio-loud AGN dominating the counts above this flux, and, it was originally thought, star-forming galaxies dominating below this flux (Condon 1984; Windhorst et al. 1985; Seymour et al. 2004). However, as suggested by Jarvis \& Rawlings (2004), radio quiet AGN will start to contribute at faint radio fluxes, and, indeed, recent observations find that AGN contribute $\sim 50 \%$ of the radio population at the $\sim 50 \mu \mathrm{Jy}$ level (Simpson et al. 2006; Seymour et al. 2008; Smolčić et al. 2008). To estimate the radio-flux due to the nuclear emission from the AGN, we use the result of Terashima \& Wilson (2003) who showed that for local radio-quiet Seyfert galaxies and quasars, $\nu L_{\nu}(5 \mathrm{GHz})=$ $10^{-5} L_{2-10} \mathrm{keV}$. Combining this result with the upper-limits for the X-ray luminosities plotted in Fig. 6 results in a distribution of radio flux due to the AGN itself (dashed histogram in Fig. 9). To calculate the $1.4 \mathrm{GHz}$ flux, a flat $\alpha=0$ spectrum was assumed, typical of the core nuclear emission from an AGN (Kellermann \& Owen 1988). We find that the AGN radio flux at $z=0.8$ are predicted to be only a factor of $\sim 3$ lower than the starburst flux, indicating that confusion between AGN and star-formation will be a factor in studying this population. However, if the data are available, methods such as spectral indices, radio morphology and radio-to-midIR flux ratios have been shown to successfully discriminate between the two possible origins (e.g., Donley et al. 2005; Seymour et al. 2008).

As noted above, both $24 \mu \mathrm{m}$ and $1.4 \mathrm{GHz}$ observations of distant AGN will have difficulty distinguishing the presence of a nuclear star-forming disk from the AGN emission. The starburst SEDs show a wide range of emission because the star-formation occurs over a broad range of temperatures. Therefore, it is useful to predict how these starbursts disks may appear in the far-IR, especially with the imminent launch of the Herschel Space Observatory. Figure 10 plots the

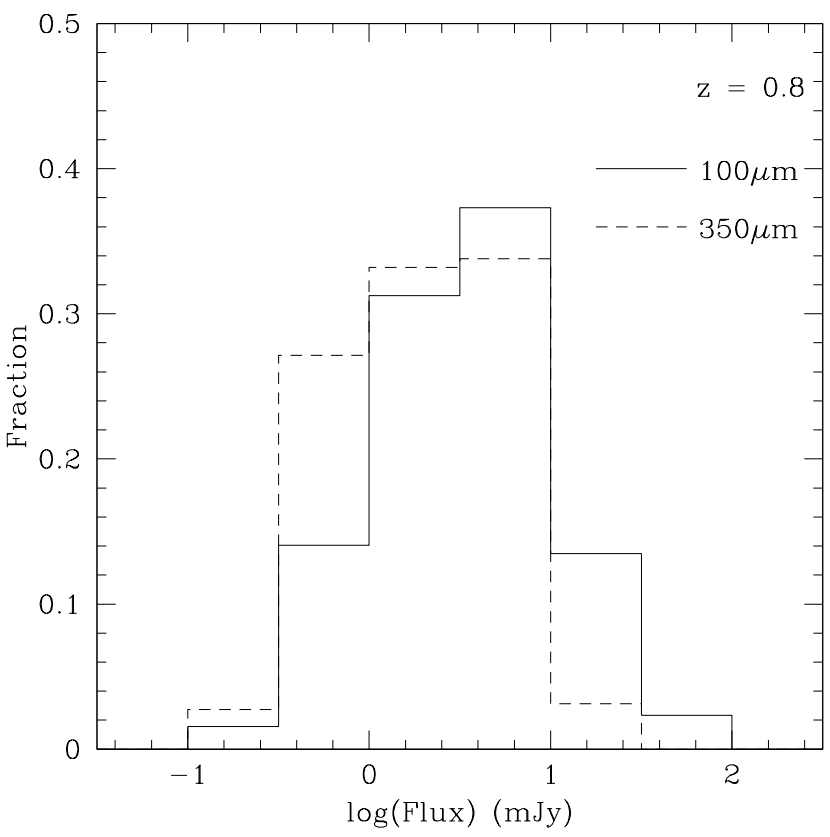

FIG. 10.- The solid histogram plots the distribution of $100 \mu \mathrm{m}$ flux predicted from the starburst disk models at $z=0.8$. The dashed line plots the same results at $350 \mu \mathrm{m}$.

$100 \mu \mathrm{m}$ and $350 \mu \mathrm{m}$ flux distributions from our starburst models at $z=0.8$. Again, since these predictions are derived from the SED calculated using eq. 1, they should be considered as upper-limits. Both of these distributions predict fluxes $\sim$ 1-10 mJy. The models of Ballantyne et al. (2006b) were employed to estimate the contribution from an AGN heated torus at these wavelengths. At both these wavelengths, the torus emission was predicted to be over an order of magnitude smaller. Those calculations assumed a relatively compact geometry for the AGN absorber, it is possible a more extended Compton-thick absorber could produce greater far-IR emission, but we defer a calculation of such a scenario until later work.

Two Herschel key projects are the PACS Evolutionary Probe $^{3}$ that has a planned sensitivity limit at $100 \mu \mathrm{m}$ of $1-$ $6 \mathrm{mJy}$. At $350 \mu \mathrm{m}$ the Herschel Multi-tiered Extragalactic Survey $^{4}$ (HerMES) predicts a sensitivity of 10-20 mJy. Thus, we predict that the PACS Evolutionary Probe survey should detect these starburst disks associated with hard X-ray sources. The HerMES survey will likely only detect the very rare high star-forming or low-redshift sources.

To summarize, the nuclear starburst disks proposed in this paper may reveal themselves to observers in multiple ways. They should have already been detected in the mid-IR by Spitzer surveys, but the predicted fluxes are of the same order as those from AGN heating of a non-star-forming torus, leading to the familiar IR spectral decomposition problem. At $1.4 \mathrm{GHz}$, the starburst disks are predicted to be slightly fainter than the deepest current radio surveys. Thus, as already indicated from previous work (Bauer et al. 2002), the fraction of radio/X-ray matches should increase as surveys probe to fainter flux levels. Unfortunately, the predicted radio flux levels are comparable to what is expected from the cen-

\footnotetext{
3 http://www.mpe.mpg.de/ir/Research/PEP/

${ }^{4}$ http://astronomy.sussex.ac.uk/ sjo/Hermes/
} 
tral radio-quiet AGN. Determining the radio spectral index or radio-to-IR flux ratios are two promising techniques that have been shown to distinguish between star-formation and nuclear activity in radio sources (e.g., Seymour et al. 2008). Finally, the cleanest test may occur in the far-IR, where the contamination from AGN heating is expected to be minimized. Figure 10 shows that these disks should be easily detectable by the planned Herschel surveys at $100 \mu \mathrm{m}$. The question then arises: if star-formation is detected in these AGN host galaxies, is there a way to determine if it is originating in the nucleus? We address this question in the next section.

\section{DISCUSSION}

\subsection{Distinguishing Between Nuclear and Galactic Starbursts}

In the previous section, some of the observational properties of the nuclear starburst disks were explored, and we showed that they should be detectable at both radio and IR wavelengths. However, at the redshifts of interest $(z \sim 1)$, it is more than likely that star-formation will be ongoing at galactic scales within the AGN host galaxy. Thus, unless the galaxy is resolved on a kpc scale or less, any observational detection of star-formation signatures from an AGN host galaxy may be ambiguous with regard to its location.

Yet, there is a key difference between the nuclear starbursts presented here and the more usual star-formation that is ongoing on large scales. As described in Sections 2.1 and 3.1 the crucial pc-scale burst of star-formation only occurs when the central temperature of the disk exceeds the dust sublimation temperature. This region of the disk does not reach these temperatures by heating by an AGN, but rather as result of the increasing density required by a star-forming $Q=1$ disk in a black hole potential. Thus, in contrast to typical Galactic star-forming regions, these models predict significant starformation from a region with very hot dust emission, but not AGN heated dust. Therefore, if such nuclear starbursts are common, there should be more near-IR or $24 \mu \mathrm{m}$ flux than expected from just pure AGN heating. As mentioned above, if this is true then it may complicate AGN selection based on power-law like or hot mid-IR colors (e.g., Donley et al.2007).

There may be evidence for this effect already in the literature. Several observations of both nearby and highredshift AGN have shown that the ratio of the absorptioncorrected $L_{X}$ to a near- or mid-IR luminosity is relatively constant with column density $N_{\mathrm{H}}$ (Lutz et al. 2004; Rigby et al. 2006; Horst et al. 2008). This is not expected from simple AGN unification and torus models where the observed midIR luminosity is predicted to depend significantly on $N_{\mathrm{H}}$ (Pier \& Krolik 1993). Evidently, there is still hot dust emission observed from AGN at all column densities. This fact has been used to argue that the AGN torus should be clumpy (Horst et al. 2008), but it is also consistent with a nuclear starburst disk around the AGN. To show this we plot in Figure 11 the distribution of the $L_{2-10 \mathrm{keV}} / \nu L_{\nu}(6 \mu \mathrm{m})$ ratio for the starburst models. This histogram should only be taken as indicative, since both the X-ray and IR luminosities are upperlimits. However, taking the results at face-value, we find that the peak of the distribution is very similar to the values observed by Lutz et al. (2004) and Rigby et al. (2006). Interestingly, the AGN torus models of Ballantyne et al. (2006b) were not able to reproduce these ratios, as they were not able to produce a sufficient near-IR luminosity. While Lutz et al. (2004) argued that they corrected their data for star-formation

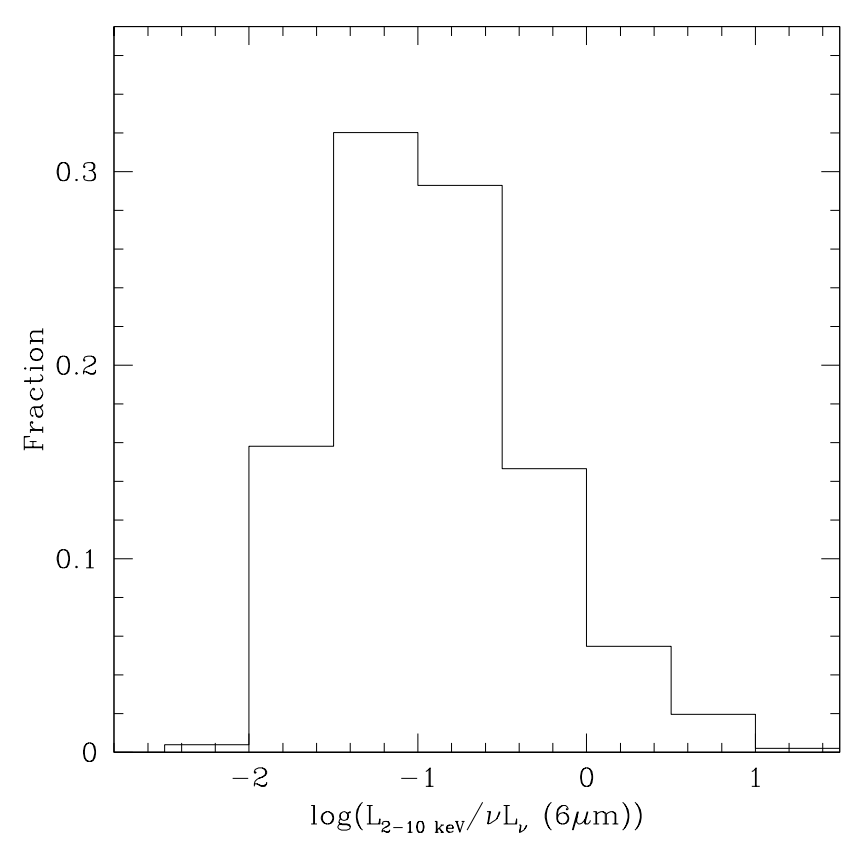

FIG. 11.- Histogram of the estimated $L_{2-10 \mathrm{keV}} / \nu L_{\nu}(6 \mu \mathrm{m})$ ratio predicted for the nuclear starburst models. They peak in the exact same range as the observations from both local and $z \sim 1$ AGN (Lutz et al. 2004; Rigby et al. 2006).

based on PAH emission, if the starburst is produced in these hot pc-scale regions, the PAH emission may be overwhelmed (Desai et al.2007) and the star-formation rate underestimated. Thus, nuclear star-formation may be another mechanism to explain the ubiquitous presence of hot dust emission even at large column densities.

\subsection{Obscuration as a Function of AGN Luminosity}

Another interesting aspect of AGN phenomenology is the observed decrease in the fraction of obscured AGN with increasing AGN luminosity (Ueda et al. 2003; La Franca et al. 2005; Akylas et al. 2006; Treister et al. 2008). This observation has been most commonly explained by the receding torus model (Lawrence 1991; Simpson 2005) which argues that the expansion of the dust sublimation radius with luminosity will push back the torus to larger radii and therefore decrease its covering factor. In this paper, we propose that the AGN obscuration is dominated by a nuclear starburst at a distance of $\sim 1$ pc. In this section, we investigate if this model can also provide an explanation for the variation of the Type 2/Type 1 ratio with luminosity.

Figure 12 plots how two of the starburst parameters depend on the estimated X-ray luminosity. The left panel of the figure plots the distribution of the maximum SFRs found in the model starburst disks. The blue and red hatched regions denote the contributions from models with $42<$ $\log \left(L_{X} /\right.$ erg s$\left.^{-1}\right)<43.5$ and $44<\log \left(L_{X} /\right.$ erg s $\left.^{-1}\right)<$ 44.5 , respectively. This plot shows that the most luminous AGN are associated with the most intense star-formation, consistent with recent observational suggestions (Shi et al. 2007; Watabe et al. 2008). In contrast, the lowest luminosity AGN are predicted with starburst disks with only modest star-formation rates. The right panel of Figure 12 plots the distribution of the input angular momentum parameter $m$. In this case, the dotted and dashed line denote the contribu- 


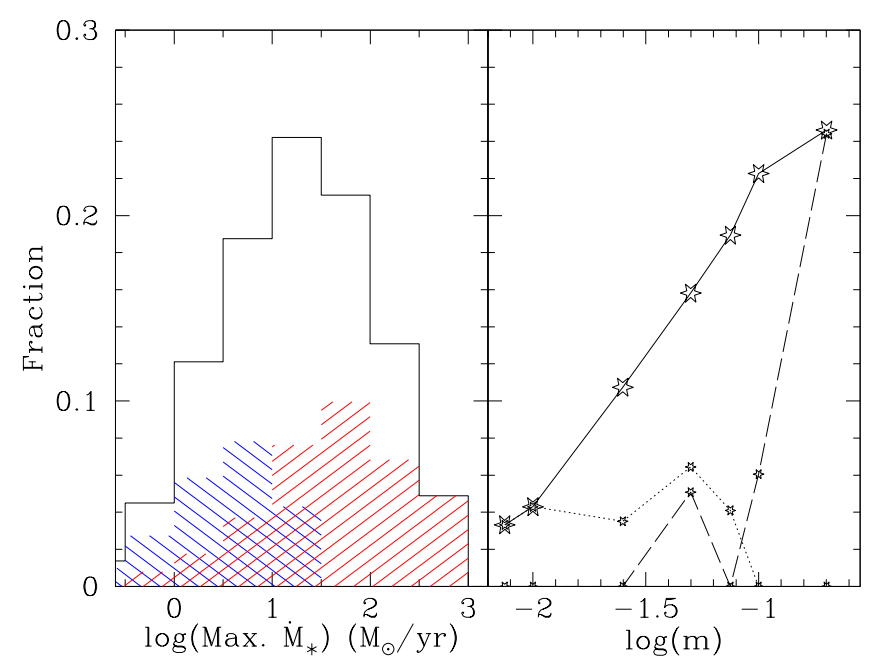

FIG. 12.- (Left) The solid histogram plots the distribution of maximum SFRs seen in the models (as seen in Fig. 4). The blue and and red hatched regions denote the contributions from models with $42<\log \left(L_{X} / \mathrm{erg} \mathrm{s}^{-1}\right)<$ 43.5 and $44<\log \left(L_{X} / \mathrm{erg} \mathrm{s}^{-1}\right)<44.5$, respectively. (Right) The solid line plots the distribution of the angular momentum parameter $m$ found in the 'successful' starburst disk models (as seen in Fig. 3. c)). The dotted and dashed lines denote the contributions from models with $42<$ $\log \left(L_{X} /\right.$ erg s$\left.^{-1}\right)<43.5$ and $44<\log \left(L_{X} /\right.$ erg s$\left.^{-1}\right)<44.5$, respectively.

tions from models with $42<\log \left(L_{X} / \mathrm{erg} \mathrm{s}^{-1}\right)<43.5$ and $44<\log \left(L_{X} / \mathrm{erg} \mathrm{s}^{-1}\right)<44.5$, respectively. As with the maximum SFRs, these two ranges of X-ray luminosities are separated in the diagram with large values of $m$ only associated with luminous AGN. Similarly, starburst disks with weak angular momentum transport (i.e., low values of $m$ ) seem to only be able to feed a low-luminosity AGN.

These results can be used to posit an explanation for the observed decrease in AGN obscuration fraction with luminosity. Recall that in this starburst disk model there is a competition between star-formation and gas accretion through the disk. If there is intense star-formation within the disk then this reduces the gas supply available to fuel a black hole. A circumnuclear starburst will therefore more likely be associated with both a lower-luminosity and obscured AGN. As the luminosity of the AGN increases, it is necessary to also increase the gas supply onto the black hole. This could be done, for example, by more efficient angular momentum transport in the starburst disk (Fig. 12). While higher luminosity AGN can be accompanied by very intense nuclear starbursts that may also obscure the AGN, such bursts will likely be shortlived. More luminous AGN, such as quasars, are unlikely to be fueled through these starburst disks, because circumnuclear star-formation would consume the necessary gas supply. The obscuration responsible for Type 2 quasars would then be generated at larger, galactic scales as a result of the powerful events shaping the host galaxy (e.g., Hopkins et al.|2005).

This starburst scenario has an advantage over alternative mechanisms that rely on AGN radiation to produce an obscuring medium (e.g., Krolik 2007; Chang et al. 2007). In these situations, more obscuration is predicted with a larger AGN luminosity, as the vertical pressure support of the obscuring medium is directly related to the intercepted AGN luminosity. A significant outflow will need to develop in order to limit the covering factor. While it is clear that a more detailed model of nuclear starburst disks is required to make quantitative predictions, we find that, at least qualitatively, identifying a pc-scale starburst as the origin of the obscuration for some AGN can provide a natural explanation for the observed decrease in the fraction of Type 2 AGN with luminosity.

\subsection{Torques and Angular Momentum Transport}

One of the most important parameters in determining whether a circumnuclear starburst can both feed and obscure an active nucleus is the efficiency of angular momentum transport through the disk. In this paper we have assumed that a global torque, such as a bar or spiral waves, operates on the starburst and removes angular momentum. We further assume that the resulting accretion rate through the disk can be sustained at a Mach number $m$. The results of Sect. 3 show that the likelihood for the formation of a pc-scale starburst that could obscure an AGN is very sensitive to $m$, with values of $0.1-0.2$ being most favorable, but lower values are certainly possible, especially for lower-luminosity AGN. Goodman (2003) argues that spiral waves in an accretion disk could cause inflow with $m \sim 0.1$, but it is not clear whether or not such global coherent structures will be common for these disks. Some insight may be gleaned from the study of gas flows in galactic nuclei (see Wada 2004 for a recent review). High-resolution simulations of the gas dynamics in a nuclear potential are now regularly finding coherent structures such as bars or spirals in the gas flows (Englmaier \& Shlosman 2004; Maciejewski 2004). These structures do remove angular momentum and funnel material to the galactic center, although the Mach number is not specified. These gas flows may ultimately become turbulent which, as argued by Wada \& Norman (2002), may also cause further gas accretion.

Alternatively, a local viscosity mechanism, such as the magneto-rotational instability (Balbus \& Hawley 1998), may operate in the starburst disk. To determine how this different angular transport process might affect the development of a pc-scale starburst, calculations of starburst disks were run with a simple $\alpha$ viscosity prescription (Shakura \& Sunyaev 1973; see also Thompson et al. 2005). For simplicity, a value of $\alpha=0.3$ was considered with all the other parameters $\left(M_{\mathrm{BH}}, R_{\text {out }}, f_{\text {gas }}\right.$, and the dust-to-gas factor) varying as before. Fifty percent of the 180 models generated a pcscale starbursts with the parameters exhibiting very similar trends to the ones shown in Fig. 3. However, as found by Thompson et al. (2005), this local viscosity does not move material inward fast enough, and the resulting mass accretion rate remaining to fuel the black hole is much smaller in this scenario than in the global torque models described above. In fact, over half of the 'successful' $\alpha$-disk models resulted in AGN with $L_{\text {bol }} / L_{\text {Edd }}<0.002$. This accretion rate is far too low to fuel the obscured AGN at $z \sim 1$ discovered by the deep $\mathrm{X}$-ray surveys.

We conclude that these relatively simple models suggest that in order for starburst disks to be a common source of AGN obscuration and fueling, an efficient angular momentum transport mechanism must operate, and a global structure, such as a bar or spiral arms, is most likely needed to provide such a mechanism.

\subsection{Starburst Disks and Unobscured AGN}

The focus of this paper has been on the properties of model starburst disks that may potentially both obscure and feed an 
AGN. Only $41 \%$ of the model disks satisfied the selection criterion (see Sect. 2.2). Here, we discuss the properties of the remaining $59 \%$ of models that did not meet the imposed conditions. By construction, these 748 starbursts disks do not have a region where the central temperature surpasses the dust sublimation temperature and produces an inner parsec-scale starburst. Therefore, the photosphere of these disks is always thin and will not obscure any central AGN.

The vast majority of this population of starburst disks are unable to fuel a central black hole at rates necessary to produce a luminous AGN. In fact, $54 \%$ of these disks would only produce an AGN with $L_{\mathrm{bol}} / L_{\mathrm{Edd}}<10^{-3}$ and $75 \%$ with $L_{\text {bol }} / L_{\text {Edd }}<10^{-2}$. These models are ones where $\tau_{*}<\tau_{\text {adv }}$ and so nearly all the gas in the disk is converted to stars at distances $\sim R_{\text {out }}$. The SFRs are within an order of magnitude to those plotted in Fig. 4 , but are in the cold, outer regions of the disk. Observationally, such systems would appear as nuclear starbursts within an inactive galaxy.

Interestingly, $25 \%$ of these 'unsuccessful' starburst disks, or 188 overall, can fuel an AGN sufficient to result in $L_{\text {bol }} / L_{\text {Edd }}>10^{-2}$. These are models where the central temperature rises toward small radii, but just does not obtain the sublimation temperature. There is no pc-scale burst of starformation, but star-formation does occur at modest rates over the entire extent of the disk. Observationally, these models would manifest themselves as unobscured, or Type 1, AGN. However, this class of starburst disks exists in a very small region of the explored parameter space, indicating that very precise conditions are required in order to result in $\tau_{*} \lesssim \tau_{\text {adv }}$. This implies that if a starburst disk is a viable mechanism to fuel the central black hole, an obscured AGN in more likely to be produced than an unobscured one. This conclusion agrees with the observed ratios of obscured to unobscured Seyfertlike AGN at $z \sim 1$ (e.g., Barger et al. 2005; Gilli et al. 2007).

Collecting together the results of all the starburst disk models reveals a possible AGN evolutionary scenario. The results of Sect. 3.1 show that obscured accretion will most likely occur at large values of $f_{\text {gas }}$. For small values of $f_{\text {gas }}$, the most likely outcome of a starburst disk will be to turn the gas into stars at large radii and an inactive central black hole. Thus, galactic nuclei may spend a large fraction of their history with small amounts of ongoing nuclear star-formation and a dormant black hole. However, processes at larger radii associated with the assembly of the host galaxy will stochastic send gas-rich material toward the nucleus. This material would have a large $f_{\text {gas }}$ and, upon passing through the starburst disk, would result in an obscured, luminous AGN. The gas supply would slowly be eroded, dropping $f_{\text {gas }}$ and removing the inner parsec-scale starburst, revealing an unobscured AGN. Finally, the gas fraction would drop to low enough levels to cut off the fueling of the black hole, and the system would return to producing stars at large radii until the remaining gas is exhausted. While speculative, this scenario may be a common occurrence during a stochastic phase of galaxy assembly.

\section{CONCLUSIONS}

In this paper we proposed that nuclear starburst disks may be an important contributor to AGN obscuration, especially at $z \sim 1$ where there is a large population of obscured AGN. The analytic model of starburst disks developed by Thompson et al. (2005) was used to explore the properties of such disks and determine if they were a viable and general method to obscure accreting black holes. We found that a range of conditions can produce potentially AGN obscuring bursts of star-formation on pc scales. These include a high dust-to-gas ratio and a relatively small size scale of the disk, both of which seem to be consistent with current constraints. The pc-scale bursts of star-formation are different from traditional star-forming regions since they are produced in a region with a temperature $\sim 1000 \mathrm{~K}$. This region is not heated by an AGN, but is a result of the assumption of a $Q=1$ stable star-forming disk in a black hole potential. Thus, the observational signature of these nuclear star-forming regions will be additional warm/hot dust emission above what is required by AGN heating. This effect may be an explanation for the observed $L_{2-10 \mathrm{keV}} / \nu L_{\nu}(6 \mu \mathrm{m})$ ratios seen from both local and high-redshift AGN. In addition, the competition between starformation and gas accretion results in a natural explanation for the decrease in the fraction of obscured AGN with luminosity. As an AGN-obscuring nuclear starburst will consume gas that may have been destined for accretion onto a black hole, this model predicts that nuclear starbursts should be closely associated with lower luminosity AGN. In contrast, high luminosity AGN require a significant gas supply, so a nuclear starburst would be an hindrance to fueling the black hole.

The observational signatures of these disks may be most easily found in future deep radio and far-IR surveys. Assuming the radio-IR correlations hold for the circumnuclear starbursts, the expected $1.4 \mathrm{GHz}$ fluxes from these disks are $\sim 10 \mu \mathrm{Jy}$ at the redshifts where the fraction of obscured AGN is near its peak. The $100 \mu \mathrm{m}$ fluxes are predicted to be $\sim 1-$ $10 \mathrm{mJy}$, which will be detectable in future Herschel surveys.

Nuclear starbursts may prove to be a compelling method of studying the symbiotic relationship between black holes and their host galaxies in the era of rapid evolution of both populations. While qualitatively interesting, this model needs to be explored further to make robust quantitative predictions. A proper estimation of the vertical structure of the disk is required to estimate covering fractions. Future work should also include incorporating the feedback from the central AGN. Another important future question is to determine the nature and observable properties of the starburst remnants.

A careful reading of the paper by the anonymous referee is gratefully acknowledged. The author is indebted to T. Thompson for very helpful discussions and advice. DRB is supported by the University of Arizona Theoretical Astrophysics Program Prize Postdoctoral Fellowship.

\section{REFERENCES}

Akylas, A., Georgantopoulos, I., Georgakakis, A., Kitsionas, S. \& Hatziminaoglou, E., 2006, A\&A, 459, 693

Alonso-Herrero, A. et al., 2006, ApJ, 640, 167

Antonucci, R., 1993, ARA\&A, 31, 473

Appleton, P.N. et al., 2004, ApJS, 154, 147

Arav, N. et al., 2007, ApJ, 658, 829

Bach, U. et al., 2007, A\&A, 464, 175

Balbus, S.A., 2003, ARA\&A, 41, 555

Balbus, S.A. \& Hawley, J.F., 1998, Rev. Mod. Phys., 70, 1

Ballantyne, D.R. \& Papovich, C., 2007, ApJ, 660, 988
Ballantyne, D.R., Weingartner, J.C. \& Murray, N., 2003, A\&A, 409, 503

Ballantyne, D.R., Everett, J.E. \& Murray, N., 2006a, ApJ, 639, 740

Ballantyne, D.R., Shi, Y., Rieke, G.H., Donley, J.L., Papovich, C. \& Rigby, J.R., 2006b, ApJ, 653, 1070

Ballo, L. et al., 2007, ApJ, 667, 97

Barger, A.J., Cowie, L.L., Brandt, W.N., Capak, P., Garmire, G.P.,

Hornschemeier, A.E., Steffan, A.T. \& Wehner, E.H., 2002, AJ, 124, 1839

Barger, A.J., Cowie, L.L., Mushotzky, R.F., Yang, Y., Wang, W.-H., Steffen, A.T. \& Capak, P., 2005, AJ, 129, 578

Barger, A.J., Cowie, L.L. \& Wang, W.-H., 2007, ApJ, 654, 764 
Barmby, P. et al., 2006, ApJ, 642, 126

Barvainis, R., 1987, ApJ, 320, 537

Bauer, F.E., Alexander, D.M., Brandt, W.N., Hornschemeier, A.E., Vignali, C., Garmire, G.P. \& Schneider, D.P., 2002, AJ, 124, 2351

Bell, E.F., 2003, ApJ, 586, 794

Beswick, R.J., Muxlow, T.W.B., Thrall, H., Richards, A.M.S. \& Garrington, S.T., 2008, MNRAS, 385, 1143

Brand, K. et al., 2006, ApJ, 644, 143

Chang, P., Quataert, E. \& Murray, N., 2007, ApJ, 662, 94

Cid Fernandes, R.J. \& Terlevich, R., 1995, MNRAS, 272, 423

Condon, J.J., 1984, ApJ, 284, 44

Crenshaw, D.M., Kraemer, S.B. \& George, I.M., 2003, ARA\&A, 2003, 41

Davies, R.I., Mueller Sánchez, F., Genzel, R., Tacconi, L.J., Hicks, E.K.S., Friedrich, S. \& Sternberg, A., 2007, ApJ, 671, 1388

De Luca, A. \& Molendi, S., 2004, A\&A, 419, 837

Desai, V. et al., 2007, ApJ, 669, 810

Di Matteo, T., Springel, V. \& Hernquist, L., 2005, Nature, 533, 604

Donley, J.L., Rieke, G.H., Rigby, J.R. \& Pérez-González, P.G., 2005, ApJ, 634,169

Donley, J. L., Rieke, G.H., Pérez-González, P.G., Rigby, J.R. \& Alonso-Herrero, A., 2007, ApJ, 660, 167

Dullemond, C.P. \& van Bemmel, I.M., 2005, A\&A, 436, 47

Elvis, M., Wilkes, B.J., McDowell, J.C., Green, R.F., Bechtold, J., Willner, S.P., Oey, M.S., Polomski, E. \& Cutri, R., 1994, ApJS, 95, 1

Englmaier P. \& Shlosman I., 2004, ApJ, 617, L115

Fabian, A.C., 1999, MNRAS, 308, L39

Fabian, A.C., Barcons, X., Almaini, O. \& Iwasawa, K., 1998, MNRAS, 297, L11

Fabian, A.C., Vasudevan, R.V. \& Gandhi, P., 2008, MNRAS, 385, L43

Ferguson, J.W., Heffner-Wong, A., Penley, J.J, Barman, T.S. \& Alexander, D.R., 2007, ApJ, 666, 261

Ferrarese, L. \& Merritt, D., 2000, ApJ, 539, L9

Förster Schreiber, N.M., Genzel, R., Lutz, D., Kunze, D. \& Sternberg, A., 2001, ApJ, 552, 544

Franceschini, A., Hasinger, G., Miyaji, T. \& Malquori, D., 1999, MNRAS, 310, L5

Franceschini, A. et al., 2005, AJ, 129, 2074

Goodman, J., 2003, MNRAS, 339, 937

Graciá-Carpio, J., García-Burillo, S., Planesas, P., Fuente, A. \& Usero, A., 2008, A\&A, 479, 703

Gebhardt, K., et al., 2000, ApJ, 539, L13

Georgakakis, A., Hopkins, A.M., Afonso, J., Sullivan, M., Mobasher, B. \& Cram, L.E., 2004, MNRAS, 354, 127

Gilli, R., Comastri, A. \& Hasinger, G., 2007, A\&A, 463, 79

González Delgado, R.M., Heckman, T. \& Leitherer, C., 2001, ApJ, 546, 845

Hamann, F. \& Ferland, G.J., 1999, ARA\&A, 37, 487

Hawley, J.F. \& Krolik, J.H., 2006, ApJ, 641, 103

Ho, L.C., 1999, ApJ, 516, 672

Ho, L.C., Filippenko, A. \& Sargent, W.L.W., 1997, ApJ, 487, 568

Hönig, S.F., Beckert, T., Ohnaka, K. \& Weigelt, G., 2006, A\&A, 452, 459

Hopkins, A.M., 2004, ApJ, 615, 209

Hopkins, P.F., Hernquist, L., Martini, P., Cox, T.J., Roberston, B., Di Matteo, T. \& Springel, V., 2005, ApJ, 625, L71

Horst, H., Gandhi, P., Smette, A. \& Duschl, W.J., 2008, A\&A, 479, 389

Ibar, E. et al., 2008, MNRAS, 386, 953

Jaffe, W. et al., 2004, Nature, 429, 47

Jarvis, M.J. \& Rawlings, S., 2004, New Astronomy Reviews, 48, 1173

Kauffmann, G. \& Haehnelt, M., 2000, MNRAS, 311, 576

Kawakatu, N. \& Wada, K., 2008, ApJ, in press (arXiv:0803.2271

Kellermann, K.I. \& Owen, F.N., 1988, in Galactic and Extragalactic Radio Astronomy, ed. G.L. Verschuur \& K.I. Kellermann (2d ed.; New York: Springer), 563

Khachikian, E.Ye. \& Weedman, D.W., 1974, ApJ, 192, 581

Kim, M., Ho, L.C. \& Im, M., 2006, ApJ, 642, 702

Krolik, J.H., 2007, ApJ, 661, 52

Lacy, M. et al., 2004, ApJS, 154, 166

Lacy, M., Sajina, A., Petric, A.O., Seymour, N., Canalizo, G., Ridgway,

S.E., Armus, L. \& Storrie-Lombardi, L.J., 2007, ApJ, 669, L61

La Franca, F. et al., 2005, ApJ, 635, 864

Lawrence, A., 1991, MNRAS, 252, 586

Levenson, N.A., Weaver, K.A. \& Heckman, T.M., 2001, ApJ, 550, 230

Lutz, D., Maiolino, R., Spoon, H.W.W. \& Moorwood, A.F.M., 2004, A\&A, 418,465

Lynden-Bell, D, 1969, Nature, 223, 690

Maggorian, J. et al., 1998, AJ, 115, 2285

Maiolino, R. \& Rieke, G.H., 1995, ApJ, 454, 95
Maciejewski, W., 2004, MNRAS, 354, 892

Melia, F. \& Falcke, H. 2001, ARA\&A, 39, 309

Miller, J.M., Raymond, J., Reynolds, C.S., Fabian, A.C., Kallman, T.R. \& Homan, J., 2008, ApJ, in press (arXiv:0802.2026)

Murray, N., Quataert, E. \& Thompson, T.A., 2005, ApJ, 618, 569

Nagao, T., Marconi, A. \& Maiolino, R., 2006, A\&A, 447, 157

Nenkova, M., Ivezić, Ž. \& Elitzur, M., 2002, ApJ, 570, L9

Neugebauer, G., Oke, J.B., Becklin, E.E. \& Matthews, K., 1979, ApJ, 230, 79

Park, S.Q. et al., 2008, ApJ, in press arXiv:0802.1191

Pérez-González, P.G., et al., 2008, ApJ, 675, 234

Pier, E.A. \& Krolik, J.H., 1993, ApJ, 418, 673

Pringle, J.E., 1981, ARA\&A, 19, 137

Richards, A.M.S. et al., 2007, A\&A, 472, 805

Rieke, G.H., 1978, ApJ, 226, 550

Rigby, J.R. et al., 2004, ApJS, 154, 160

Rigby, J.R., Rieke, G.H., Donley, J.L., Alonso-Herrero, A. \&

Pérez-González, P.G., 2006, ApJ, 645, 115

Risaliti, G., Elvis, M. \& Nicastro, F., 2002, ApJ, 571, 234

Risaliti, G., Elvis, M., Fabbiano, G., Baldi, A. \& Zezas, A., 2005, ApJ, 623, L93

Rodríguez Zaurín, J., Tadhunter, C.N. \& González Delgado, R.M., 2008, MNRAS, 384, 875

Rovilos, E., Georgakakis, A., Georgantopoulos, I., Afonso, J., Koekemoer,

A.M., Mobasher, B. \& Goudis, C., 2007, A\&A, 466, 119

Sanders, D.B., Phinney, E.S., Neugebauer, G., Soifer, B.T. \& Matthews, K., 1989, ApJ, 347, 29

Semenov, D., Henning, Th., Helling, Ch., Ilgner, M. \& Sedlmayr, E., 2003, A\&A, 410, 611

Setti, G. \& Woltjer, L., 1989, A\&A, 224, L21

Seymour, N., McHardy, I.M. \& Gunn, K.F., 2004, MNRAS, 352, 131

Seymour, N., Dwelly, T., Moss, D., McHardy, I., Zoghbi, A., Rieke, G.,

Page, M., Hopkins, A. \& Loaring, N., 2008, MNRAS, 386, 1695

Shakura, N.I. \& Sunyaev, R.A., 1973, A\&A, 24, 337

Shi, Y., Ogle, P., Rieke, G.H., Antonucci, R., Hines, D.C., Smith, P.S., Low, F.J., Bouwman, J. \& Willmer, C., 2007, ApJ, 669, 841

Shi, J. \& Krolik, J.H., 2008, ApJ, 679, 1018

Silk, J. \& Rees, M.J., 1998, A\&A, 331, L1

Simpson, C., 2005, MNRAS, 360, 565

Simpson, C., Martínez-Sansigre, A., Rawlings, S., Ivison, R., Akiyama, M., Sekiguchi, K., Takata, T., Ueda, Y. \& Watson, M., 2006, MNRAS, 372, 741

Smolčić, V. et al., 2008, ApJS, in press (arXiv:0803.0997)

Snijders, L., Kewley, L.J. \& van der Werf, P.P., 2007, ApJ, 669, 269

Spergel, D.N., et al., 2003, ApJS, 148, 175

Stern, D., et al., 2005, ApJ, 631, 163

Terashima, Y. \& Wilson, A.S., 2003, ApJ, 583, 145

Thompson, T.A., Quataert, E. \& Murray, N., 2005, ApJ, 630, 167

Thompson, T.A., Quataert, E., Waxman, E., Murray, N. \& Martin, C.L., 2006, ApJ, 645, 186

Tozzi, P. et al., 2001, ApJ, 562, 42

Tozzi, P. et al., 2006, A\&A, 451, 457

Treister, E. \& Urry, C.M., 2006, ApJ, 652, L79

Treister, E., Krolik, J.H. \& Dullemond, C., 2008, ApJ, in press (arXiv:0801.3849)

Tremaine, S., et al., 2002, ApJ, 574, 740

Tristram, K.R.W. et al., 2007, A\&A, 474, 837

Ueda, Y., Akiyama, M., Ohta, K. \& Miyaji, T., 2003, ApJ, 598, 886

Vasudevan, R.V. \& Fabian, A.C., 2007, MNRAS, 381, 1235

Wada, K., 2004, in Coevolution of Black Holes and Galaxies, from the Carnegie Observatories Centennial Symposia, ed. L.C. Ho (Cambridge: Cambridge University Press)

Wada, K. \& Norman, C., 2002, ApJ, 566, L21

Wang, J.L., Xia, X.Y., Mao, S., Cao, C., Wu, H. \& Deng, Z.G., 2006, ApJ, 649,722

Watabe, Y., Kawakatu, N. \& Imanishi, M., 2008, ApJ, 677, 895

Wilkes, B.J., Pounds, K.A., Schmidt, G.D., Smith, P.S., Cutri, R.M., Ghosh, H., Nelson, B. \& Hines, D.C., 2005, ApJ, 634, 183

Windhorst, R.A., Miley, G.K., Owen, F.N., Kron, R.G., \& Koo, D.C., 1985, ApJ, 289, 494

Wyithe, J.S.B. \& Loeb, A., 2003, ApJ, 595, 614

Yun, M.S., Reddy, N.A. \& Condon, J.J., 2001, ApJ, 554, 803 